University of Nebraska - Lincoln

DigitalCommons@University of Nebraska - Lincoln

\title{
Nonequilibrium Thermodynamics Modeling of Coupled Biochemical Cycles in Living Cells
}

Yaşar Demirel

University of Nebraska - Lincoln, ydemirel2@unl.edu

Follow this and additional works at: https://digitalcommons.unl.edu/cbmedemirel

Part of the Chemical Engineering Commons

Demirel, Yaşar, "Nonequilibrium Thermodynamics Modeling of Coupled Biochemical Cycles in Living Cells" (2010). Yasar Demirel Publications. 7.

https://digitalcommons.unl.edu/cbmedemirel/7

This Article is brought to you for free and open access by the Chemical and Biomolecular Research Papers -- Faculty Authors Series at DigitalCommons@University of Nebraska - Lincoln. It has been accepted for inclusion in Yasar Demirel Publications by an authorized administrator of DigitalCommons@University of Nebraska - Lincoln. 
Published in the Journal of Non-Newtonian Fluid Mechanics (2010) 165: 953-972. DOI: 10.1016/j.jnnfm.2010.02.006. Copyright of published article 2010, Elsevier. http:/ / www.elsevier.com/locate/jnnfm. Used by permission. This is a manuscript copy. Partly presented in the $5^{\text {th }}$ International Workshop on Non-Equilibrium Thermodynamics and Complex Systems, Cuernavaca, Mexico, August 24-28, 2009.

\title{
Review \\ Nonequilibrium Thermodynamics Modeling of Coupled Biochemical Cycles in Living Cells
}

\author{
Yaşar Demirel \\ Department of Chemical and Biomolecular Engineering, University of Nebraska-Lincoln, Lincoln, USA; ydemirel2@unl.edu
}

\begin{abstract}
Living cells represent open, nonequilibrium, self organizing, and dissipative systems maintained with the continuous supply of outside and inside material, energy, and information flows. The energy in the form of adenosine triphosphate is utilized in biochemical cycles, transport processes, protein synthesis, reproduction, and performing other biological work. The processes in molecular and cellular biological systems are stochastic in nature with varying spatial and time scales, and bounded with conservation laws, kinetic laws, and thermodynamic constraints, which should be taken into account by any approach for modeling biological systems. In component biology, this review focuses on the modeling of enzyme kinetics and fluctuation of single biomolecules acting as molecular motors, while in systems biology it focuses on modeling biochemical cycles and networks in which all the components of a biological system interact functionally over time and space. Biochemical cycles emerge from collective and functional efforts to devise a cyclic flow of optimal energy degradation rate, which can only be described by nonequilibrium thermodynamics. Therefore, this review emphasizes the role of nonequilibrium thermodynamics through the formulations of thermodynamically coupled biochemical cycles, entropy production, fluctuation theorems, bioenergetics, and reaction-diffusion systems. Fluctuation theorems relate the forward and backward dynamical randomness of the trajectories or paths, bridge the microscopic and macroscopic domains, and link the time-reversible and irreversible descriptions of biological systems. However, many of these approaches are in their early stages of their development and no single computational or experimental technique is able to span all the relevant and necessary spatial and temporal scales. Wide range of experimental and novel computational techniques with high accuracy, precision, coverage, and efficiency are necessary for understanding biochemical cycles.
\end{abstract}

Keywords: Biological systems, Network dynamics, Stochastic equations, Nonequilibrium thermodynamics, Fluctuation theorems, Bioenergetics, Reaction-diffusion systems

Contents

1. Introduction

2. Biological systems

2.1. Some relevant experiments

2.2. Some relevant computational methods

2.3. Dynamic components

2.4. Thermodynamic constraints

3. Modeling

2.5. Self organization and energy dissipation

3.1. Deterministic models

3.2. Stochastic models

3.3. Multiscale computer simulation

3.4. Network dynamics

3.5. Molecular motors

4. Fluctuation theorems

4.1. Application to the $\mathrm{F}_{\mathrm{o}} \mathrm{F}_{1}$-ATPase molecular motor

5. Bioenergetics

4.2. Application to molecular motor kinesin

5.1. Linear nonequilibrium thermodynamics analysis of oxidative phosphorylation

5.2. Chemomechanical energy transduction

6. Reaction diffusion systems

6.1. Linear nonequilibrium thermodynamics modeling

7. Conclusions

\section{Nomenclature}

A affinity (Eq. 21)

$A^{*} \quad$ nondimensional affinity in Eqs. (63)and (64)

$c_{p} \quad$ heat capacity

$C_{i} \quad$ concentration of specie $i$

Da Damköhler number, dimensionless and defined in Eqs. (63) and (64)

$D_{i} \quad$ Diffusion coefficient of species $i$

$D_{S, e}$ effective diffusion coefficient for the substrate $\mathrm{S}, \mathrm{m}^{2} \mathrm{~s}^{-1}$

$D_{D, e}^{s, e} \quad$ coupling coefficient related to the Dufour effect, $\mathrm{J} \mathrm{m}^{2} \mathrm{~mol}^{-1} \mathrm{~s}^{-1}$

$D_{T, e}$ coupling coefficient related to the thermal diffusion (Soret effect), $\mathrm{mol} \mathrm{m}^{-1} \mathrm{~s}^{-1} \mathrm{~K}^{-1}$

E activation energy of the chemical reaction, $\mathrm{J} \mathrm{mol}^{-1}$

F Faraday constant

$\Delta H_{r} \quad$ reaction enthalpy, $\mathrm{J} \mathrm{mol}^{-1}$
G Gibbs free energy

$j \quad$ flow ratio (Eq. 46)

$J \quad$ diffusive mass flux (flow), $\mathrm{mol} \mathrm{m}^{-2} \mathrm{~s}^{-1}$

$J \quad$ conduction heat flux (flow), $\mathrm{W} \mathrm{m}^{-2}$

$J_{r}^{q} \quad$ volumetric reaction rate $\mathrm{mol} \mathrm{m}^{-3} \mathrm{~s}^{-1}$

$k_{e} \quad$ effective thermal conductivity, $\mathrm{W} \mathrm{m}^{-1} \mathrm{~K}^{-1}$

$k_{B} \quad$ Boltzmann constant

$k_{i} \quad$ rate constant for chemical reaction $i$

$K \quad$ equilibrium constant

$K_{M} \quad$ Michaelis constant

$L \quad$ characteristic half thickness, $\mathrm{m}$

Le modified Lewis number, dimensionless and defined in Eq. (64)

$L_{i k} \quad$ phenomenological coefficients

$L_{q r} \quad$ coupling coefficient between chemical reaction and heat flow, $\mathrm{mol} \mathrm{K} \mathrm{m} \mathrm{m}^{-2} \mathrm{~s}^{-1}$ 
$L_{S r} \quad$ coupling coefficient between chemical reaction and mass flow, $\mathrm{mol}^{2} \mathrm{~K} \mathrm{~J}^{-1} \mathrm{~m}^{-2} \mathrm{~s}^{-1}$

$n$ number of components

$N_{r} \quad$ number of independent reactions

$P \quad$ probability

$\mathrm{P}_{\mathrm{i}} \quad$ phosphate

$q \quad$ degree of coupling (Eq. 44)

$Q \quad$ heat

$R \quad$ gas constant

$S$ entropy

$t \quad$ time, $\mathrm{s}^{-1}$

$T$ temperature, $\mathrm{K}$

$u \quad$ internal energy

$V \quad$ total volume, $\mathrm{m}^{3}$

$W$ work

$x \quad$ thermodynamic force ratio (Eq. 46)

$X \quad$ thermodynamic force

$z \quad$ dimensionless distance; charge

Z phenomenological stoichiometry; partition function

\section{Abbreviations}

ATP adenosine triphosphate

ADP adenosine diphosphate

BST biochemical system theory

CME chemical master equation

DNA deoxyribonucleic acid

EBA energy balance analysis

FBA flux balance analysis

LNETlinear nonequilibrium thermodynamics

MCA metabolic control analysis

MM Michaelis-Menten

NESS nonequilibrium steady state

OP oxidative phosphorylation

PFK phosphofructokinase

RNA ribonucleic acid

SNT stoichiometric network thermodynamic

\section{Introduction}

The human genome project revealed that understanding the biological structures, a comprehensive description of deoxyribonucleic acid (DNA), protein and their functions is needed. The large scale biological data sets and more time sequence data generated by the human genome project [1-4] require expertise beyond traditional biology, such as chemists, physicists, mathematicians, engineers, computer scientists, and others. Consequently, the efforts in analyses of component biology and system biology as well as analyses for bridging them have attracted researchers from variety of disciplines. Often, however, the researchers emphasize their point of view in their descriptions and interpretations of important steps, and yet the standards of these descriptions, interpretations, procedures, and formulations vary from one group or lab to another and leads to some communication difficulties. Component biology is closely related to enzyme kinetics and fluctuation of single biomolecule acting as molecular motors. Systems biology, on the other hand, involves FCA functional cellular attractorscomprehensive analysis of the complex biological organization and processes in which all the components of a biological system interact functionally over time and space [510]. Systems biology has various evolving stages, such as molecular self organization, large-scale models, metabolic control analysis, and convergence of many concepts of chemistry, mathematics, thermodynamics, and molecular biology. The integrated elements of systems biology are [5,10]: (1) designed biological experiments, (2) experimental technologies at cellular level, (3) biocomputation and bioinformation for processing and presentation of large scale data sets $[1,2]$, and (4) conceptual and mathematical frameworks to analyze stochastic data and model biological cycles [11,12-19]. Data collected from experiments on genomic and metabolic scales help identifying the components and interactions in biochemical cycles and networks. With the right algorithms and approaches, such data can
Greek symbols

$\beta$ friction coefficient; thermicity group, dimensionless and defined in Eqs. (63)and (64)

$\gamma \quad$ Arrhenius group, dimensionless defined in Eqs. (63)

$\varepsilon \quad$ cross coefficient related to Soret effect, dimensionless and defined in Eqs. (63)

random force

energy conversion efficiency (Eq. 47)

dimensionless concentration defined in Eqs. (63)

cross coefficient dimensionless and defined in Eq. (64)

chemical potential

density

entropy production; cross coefficient, dimensionless and defined in Eq. (64)

dimensionless time

$\Phi \quad$ volumetric entropy generation rate, $\mathrm{W} \mathrm{m}^{-3} \mathrm{~K}^{-1}$

network potential as an emerging property (Eq. 19)

dimensionless temperature defined in Eqs. (63)

$\omega$ cross coefficient related to Dufour effect, dimensionless and defined in Eq. (64)

$\begin{array}{ll}\text { Subscripts } \\ \mathrm{b} & \text { backward } \\ \text { dis } & \text { dissipation } \\ \mathrm{D} & \text { Dufour } \\ \text { e } & \text { effective } \\ \text { ext } & \text { external } \\ \text { eq } & \text { equilibrium } \\ \mathrm{f} & \text { forward } \\ \text { opt } & \text { optimum } \\ \mathrm{q} & \text { heat } \\ \mathrm{r} & \text { reaction } \\ \mathrm{s} & \text { surface } \\ \text { sh } & \text { static head } \\ \mathrm{S} & \text { Soret } \\ \text { unc } & \text { uncoupled }\end{array}$

also help determining biochemical reaction mechanisms, which clarify the set of elementary steps, the nature of reaction intermediates, their interactions in transformations, and the rates of these transformations. The mechanisms also help understand the functional role of various molecules, control of various biochemical pathways, and kinetic modeling [19].

Living cells represent open, nonequilibrium, self organizing, and dissipative systems maintained with the continuous supply of outside and inside material, energy and information flows. Therefore, it can create and maintain pathways in which thermodynamically coupled and critically synchronized rate and transport processes of the biochemical cycles take place $[20,21]$. Here, the coupling refers that a flow occurs without or against its primary thermodynamic driving force, which may be a gradient of temperature, or a chemical potential, or an affinity. The principles of thermodynamics allow the progress of a process without or against its primary driving force only if it is coupled with another spontaneous process. This is consistent with the statement of second law, which states that a finite amount of organization may be obtained at the expense of a greater amount of disorganization in a series of coupled processes. Living systems survive as they are capable of funneling material, energy, and information into their own production and reproduction, and contribute to the pathways of autocatalytic processes of biochemical cycles, which are bounded with conservation, kinetics, and thermodynamic laws $[6,14,16]$.

The processes in molecular and cellular biological systems are stochastic in nature with varying scales of time and space $[11,17,18]$. The stochastic differential equations can be linked to both the master equation type description with explicit discrete nature of chemical processes and the Fokker-Planck equation type with continuous variables in both time and state space [11]. Therefore, the modeling 
helps to understand biological structure, perform exploring simulations, interpret and evaluate of measured data, make predictions, and help design further experiments [11,13-19].

Systems in global equilibrium with large number of molecules follow the laws of classical thermodynamics, which investigate systems with larger length and longer time scales, and less molecular detail $[26,27]$. However, living systems of biochemical cycles operate far from equilibrium and constrained by large gradients (thermodynamic forces), and utilize all means available to counter these applied gradients [6,22-25]. Fluctuations at far from equilibrium dominate the behavior of biological systems and can lead to selforganized dissipative structures, such as biochemical cycles acquiring low entropy by increasing the entropy in their environments $[6,12,22]$. At each bifurcation, existing structures trigger the process that leads to new, more complex, and more stable forms of order [6-8,22-25]. In its more general form, fluctuation theorems provide an analytical description of how irreversible macroscopic behavior evolves from time-reversible microscopic dynamics as either the system size or the observation time increases [26,27]. Therefore, fluctuation theorems bridge the microscopic and macroscopic domains, link the time-reversible and irreversible descriptions, help understanding the unique properties of microscopic and mesoscopic systems, and may serve as the basis for a theory of the nonequilibrium thermodynamics of small systems [6,26-29].

Living systems grow by adding more of the same types of pathways and develop when new pathways and networks emerge $[30,31]$. Necessary energy for growth and development comes $[21,31,32]$ from energy converters that couple input flow-force pairs with the corresponding output flow-force pairs in biochemical cycles. Molecular motors ranging in size from 2 to $10 \mathrm{~nm}$ in a thermal bath, for which fluctuations can be even larger than the mean values, converts chemical energy into useful work in the cell. Subsequent$l y$, the cell performs many biological work by utilizing the energy released by the hydrolysis of adenosine triphosphate (ATP) $[14,23,24]$. Biological work includes biosynthesis of proteins, nucleic acids, lipids and polysaccharides, ATP production, active transport and excretion of wastes. Some of the mechanical work consist of cell division and muscle contraction, while some of the electrical work involve transmission of nerve impulses to osmotic activity and firefly illumination. All these work involve complex energetic coupling, in which complexity is the number of successive levels of hierarchical structuring in a system-part to whole [24,31-37].

The self-organizing processes of living cells are linked to certain functions and are characteristically different from other dynamic organizations that do not necessarily acquire a function $[6,9,22,23]$. Consequently, biochemical cycles emerge from collective and functional efforts to devise a cyclic flow of optimal energy degradation rate, which can only be described by nonequilibrium thermodynamics $[6,21,29,31,32,36,38-53]$. For example, as a major part of organization, the thermodynamic coupling in the membranes of living cells plays major role in the respiratory electron transport chain leading to synthesizing of ATP [31].

This review starts with a general description of biological systems in which experimental and computational techniques, dynamic character, thermodynamic constraints, and self-organizing processes of biological systems are briefly discussed. Some approaches for modeling of biochemical cycles and macromolecules are reviewed with an emphasis of what they mean individually and how they are interrelated in a large variety of spatial and time scales. Nonequilibrium thermodynamics of microscopic and macroscopic biological systems are reviewed within the context of modeling self-organized dissipative systems $[22,23]$. Later, the fluctuations theorems with some specific applications for macromolecules are reviewed. Bioenergetics and linear nonequilibrium analysis of ATP production is reviewed next. Lastly, reaction-diffusion systems and some examples of linear nonequilibrium formulations are reviewed. Therefore, this review emphasizes the role of nonequilibrium thermodynamics through the formulations of coupled biochemical cycles in near and far from equilibrium, entropy production, and fluctuation the- orems. This review also emphasizes the need of a truly collective effort to understand and describe the thermodynamically coupled biological systems within the constraints of conservation, thermodynamics, and kinetics laws.

\section{Biological systems}

DNA and ribonucleic acid (RNA) are two different nucleic acids found in the cells of every living organism and have cooperating roles in the field of cell biology. DNA contains the genetic information of an organism. This information dictates how the body's cells would construct new proteins. DNA, RNA, protein and some other complex organic molecules give biological systems their unique properties, which emerge out of the interaction of the components comprising the whole system. The eight levels of living systems are cells, organs, organisms, groups, organizations, communities, societies, and supranational systems. The cells are the basic building blocks of all living systems [4].

Sequenced genomes together with gene expression data help understanding of metabolic networks [3,4], which consist of typically several hundreds of reactions that are catalyzed by enzymes. These metabolic reactions of an organism can to some extent be determined from its genome since the presence of an enzyme and the particular reaction it catalyzes can be ascertained by sequence homology analysis. A flux (flow) is assigned to each metabolic reaction to predict the behavior of the cell as a result of interactions between the different reactions that go on inside the cell [19]. Some important concepts in biological transformations are state, key state, conformational state, kinetic state, transition, trajectory, and restraint. A state characterizes chemical and physical composition, and three dimensional structure of a macromolecule or an assembly. Creation of a structural dynamics model of a macromolecular process, such as active transport with molecular motors and protein synthesis by the ribosome, requires determination of key states and their structural characterization, since the set of key states and transitions between them defines the essence of the process [54]. A trajectory is a detailed sequence of states and describes a transition between two key states, which may be stable or unstable. Conformational heterogeneity implies that multiple states exist in a single molecule or an assembly, while the kinetic heterogeneity results from different copies of the system following different transitions; for example, different parts of a protein can form and function independently. A restraint restricts geometric and dynamic properties of an assembly, such as the distance between two components, overall shape of the complex, or the time interval between two key states. A biological dynamic process is represented as a set of key states connected by transitions with associated trajectory, rate and transport information involving single macromolecule, macromolecular complexes, and biochemical cycles taking place over a wide variety of spatial and time scales $[13,17,55-59]$. Table 1 shows the spatial and time scales of biological systems. Currently, no single computational or experimental technique is able to span all the relevant and necessary spatial and temporal scales, and wide range of experimental and novel computational techniques with high accuracy, precision, coverage, and efficiency are necessary for understanding biological systems in general [11,15-17].

\subsection{Some relevant experiments}

Some of the current experimental techniques used in biological systems are [54]: (i) Time resolved small angle X-ray scattering collects a time course of the scattering profile by repeatedly exposing a sample in solution to determine the structure of intermediates in the sample [19]. (ii) Transverse relaxation optimized spectroscopy is a variant of nuclear magnetic resonance spectroscopy that can be applied to large systems. This method isolates part of the system by replacing the remaining hydrogen atoms with deuterium atoms to be monitored to measure local conformation and its changes. (iii) In pulse-chase monitored by quantitative mass spectrometry, a complex is allowed to assemble for some period, followed by a rapid dilution of nonbound proteins in solution with $\mathrm{N}^{14}$ labeled proteins. It then measures the rela- 
tive populations of the heavy and light molecules, producing an association rate estimate for accumulation in the complex. (iv) In time resolved pullouts, [60] the cells are rapidly frozen and the media is ground and is thawed. The protein is pulled out by affinity chromatography and identified with mass spectrometry or other methods. (v) In another technique, flourescent tags [61] are attached to particles and the system is observed through a microscope. When the marked particles are separated by at least tens of nanometers, the individual dyes can be located [60]. (vi) In forster resonance energy transfer spectroscopy [61], two particles are tagged with appropriate fluorophores; when the dyes are close to one another (several nanometers), they become coupled and the strength of this coupling, which depends on distance, detecs changes in distance. (vii) In optical tweezers, [62] a micron-sized polystyrene bead attached to part of the system is held in an optical trap, which can be used either to hold the bead at a specified force or to set the displacement over time. Some recent setups allow a second bead to be trapped and manipulated independently. Optical tweezers can apply forces of up to hundreds of piconewtons.

\subsection{Some relevant computational methods}

Structural modeling of a dynamic biological process usually begins with the determination of key states, their structural characterization [54], and trajectories between key states. In molecular dynamics [63] Newton's equations of motion are integrated for the atoms of the system by relying on a molecular mechanics force field to obtain a trajectory of the system with time steps on the order of femtoseconds. Parallel molecular dynamics programming can handle large systems [63]. Coarse graining and multiscale methods can extend the reachable time scales to fractions of a millisecond by representing many atoms with a single particle, using force fields derived from more detailed all-atom computations, and adding intermediate key states along the transition $[17,57]$. Computer simulations are reaching the microsecond time scale, while the stoppedflow techniques reach the millisecond time scale. In normal modes dynamics [64], the assembly is represented as a collection of points connected by springs. The local dynamics of such an object is approximated by a linear combination of a small basis set of harmonic motions, each with a characteristic frequency. The trajectory is generated by an iterative extrapolation of local dynamics. Motion planning [3] algorithms are a large family of techniques taken from robotics that search for noncolliding trajectories between two known states of the system. Stochastic path integral for coarse-grained simulations can preserve statistical characteristics of the original dynamics. With the stochastic path integral, large networks of stochastic reactions can be reduced to a set of deterministic equations [65].

There are some other methodologies to describe metabolic network study: In the Flux balance analysis (FBA), the mass conservation is built into the formulation and a linearization near a steadystate state with the given stoichiometric constraints takes place. The first eliminates redundant dynamical variables, while the second is a special case by setting the stochastic force to be zero. The FBA is easy to be implemented in practical applications, because many matured mathematically tools, such as linear programming, can be employed [3]. Metabolic control analysis (MCA) studies how a network in steady state responds to changes in fluxes without dynamics and stochastic force. Properties of the architectural structure of the metabolic network can be revealed by MCA. In Biochemical systems theory (BST) [4] the force is assumed to polynomial for an easy mathematical analysis, but the stochastic force is typically neglected, while the control theory is employed. Stoichiometric network thermodynamic theo$r y$ (SNT) explicitly incorporates thermodynamic constraints into the modeling [30] since energy and entropy play such dominant roles in metabolic network dynamics [30,66-68]. However, an integration of experimental and theoretical considerations is necessary as none of these computational techniques are always accurate, applicable on all relevant time and size scales, and capable of describing all properties of interest [54].

\subsection{Dynamic components}

Dynamic components of a given biological network are the forces (driving and transverse), the flows (fluxes), the dissipation, and the noise $[28,57,69]$. The driving force provides the necessary resource in order to start and maintain the desired function, and determines the distance from equilibrium and robustness of the network. The transverse force is responsible of relocation or translocation of the resource from one part of the network to another, the delay response, and the oscillation. The dissipation describes how the resource is consumed. Owing to dissipation, the network needs constant supply of external and internal resources. Finally, for any complex biological network, there always exists stochastic force. Both dissipative and stochastic characteristics provide the network with the unique ability to adapt to the optimal state. At the same time, the generic dynamical principles require that all the components must be bounded by the constraints and the stochastic force must be the integral part of the network $[11,70]$. With the identification of the dynamical components, final stationary distribution can be estimated from the forces, and the time scales in the network become explicit $[11,16,17]$.

\subsection{Thermodynamic constraints}

Biological systems work under mass and energy conservations as well as thermodynamic constraints arising from the second law [11,71-74]. The flux balance analysis is based on mass conservation; and the energy balance analysis (EBA) is based on the nonequilibrium network thermodynamics, which states that each internal reaction with non-zero flux must dissipate energy $[25,74]$. The FBA has proved to be useful for studying the steady-state metabolic flux inside the cell in the absence of knowledge of detailed kinetic parameters of reactions [74-76]. For a flux vector $\mathbf{J}$ to be thermodynamically

feasible, there must exist a vector $\Delta \mu(\Delta \mathrm{m}=\mathrm{m} \cdot \mathbf{S})[71]$ for which

the thermodynamic constraint is satisfied $\Delta \mathrm{m}_{j} J_{j} \leq 0$; here $\mu$ is the chemical potential and $\mathbf{S}$ is the stoichiometric matrix of metabolic chemical reactions. Furthermore, $J_{j}=0$ if only $\Delta \mu=0$. According to the second law, fluxes must flow from reactants of higher chemical potential to ones of lower chemical potential and the entropy of the reaction is always non-decreasing [21,76]. Nigam and Liang [72,73] provided an algorithm for flux and energy balance analyses that perturb the metabolic network to find a thermodynamically feasible solution. If one deletes reactions corresponding to zero fluxes, the metabolic network is modified, and this may lead to an optimal thermodynamically feasible solution. It should, however, be noted that the FBA and EBA may still not able to constrain the metabolic network completely and lead to an infinity of flux and change in chemical potential vectors [72]. More realistic bounds on the values of fluxes are required to further constrain the system by studying the biochemistry of several pathways. A more complete formulation could make the change in chemical potential for each internal reaction more interpretable and comparable to experiment.

\subsection{Self organization and energy dissipation}

In 1960s, Prigogine $[6,48,49]$ formalized the nature of dynamic self organization as emergence of order in systems with as an ability to dissipate the energy gradients effectively. As Karsenti [23] notes that the importance of self-organizing processes of biochemical cycles was recognized in 1980s and 1990s, and gain momentum only recently $[22,35,37,56]$.

Mostly, cell organization depends on the self-assembly processes, which are at thermodynamic equilibrium, and hence do not involve energy dissipation. In living cell, however, self-organization also occurs by dissipating the ATP. The dynamic order of biological systems results from variety of dynamic interactions as some spontaneous processes thermodynamically couple with processes that require energy from outside hence the total entropy production for the whole system becomes positive, which is required by the second law of thermodynamics. Therefore, some processes for certain functions, which cannot proceed on their own, become possible through 
the coupling, and consequently order emerges for the system. For example, self organization of microtubules and cell cortex feedback on each other to generate a self-organized dynamic cell shape [7$9,23,77]$. Also many enzymatic biological cycles oscillate that lead to dynamic instability of collective binding and unbinding and hence to temporal patterns $[18,23]$, while the spindle of the nucleus represents a spatial self-organization. This indicates that there is ensemble of systems that can couple dynamically to reach a functional steady state $[8,23,35,37]$. Table 2 summarizes some important principles, concepts, and examples of self-organization in biological systems.

As Karsenti states [23], the character of biological systems as selforganized biochemical cycles forces us to focus on collective behaviors and principles rather than on single process or molecule, and use physical sciences and computer simulations to understand these coupled dynamic systems. Self organization in biological systems occurs at far from global equilibrium on thermodynamic branch shown in Figure 1. Table 2 and Fig 1 show that the distance from global equilibrium emerges as a thermodynamic factor controlling the behavior of systems. Only after a critical distance, a biological system bifurcates due to enhanced fluctuations leading to multiple states among which a self-organized dissipative biochemical cycle emerges as a functional stable state.

\section{Modeling}

Biological systems have evolved pathways, molecular structures and machinery at the nanoscale to power sophisticated and synchronized cell functioning. However, current tools usually do not measure these entities directly but infer their presence using prior knowledge of their presence as most of our knowledge of biological systems is descriptive and qualitative in nature [58]. Some major challenges in formulation and analysis of biochemical cycles are stochastic modeling, functional cellular attractors, multiple spatial and time scales, and thermodynamics of open and nonequilibrium systems $[11,13-17,55,38,93]$. Some chemical systems inside a cell, such as signaling networks involving transcription regulation, protein phosphorylation, and ATPases, often involve a small number of molecules of one or more of the reactants. Thus, the traditional methods of describing concentration changes with ordinary differential equations (ODEs) and the law of mass action [34] become insufficient. A Markov chain (or master equation) model accounts for the discrete, probabilistic nature of the chemical reactions at the molecular level, but can be difficult to analyze. Diffusion (Fokker-Planck) approximations can match the solution to the master equation in the thermodynamic limit for some finite time. However, unless the steady state is unique in the macroscopic description, the two models can disagree in the infinite time limit [38]. Microscopic simulations have validated the master equation as the most accurate description of a reactive process $[11,13]$. In terms of the chemical master equation (CME) formulation [38,94,95], each stable steady state of the deterministic model corresponds to a peak, while an unstable steady state corresponds to a saddle in the stationary probability distribution. These states are called as functional cellular attractors (FCA) in which the system is most likely to be found to perform its function [38]. The CME and Fokker-Planck descriptions can yield conflicting answers to the question how long the system remains in each of the FCA, while Schlogl's model exhibits multiple time scales; a fast scale where the system relaxes to one of the FCAs, and a slow scale over which the system transitions from one FCA to another [96]. Focusing on measurement technologies will rely on an iterative relationship with computational modeling approaches that are capable of dealing with the gradients and discontinuities of biological systems. New modeling approaches, on the other hand, will be required to represent the nonlinearity, adaptive, and collective behavior of biological systems by using the responses as assets of a biological network rather than simply the programmed responses of cells [53-59].

Biochemical nonequilibrium reaction systems operate with fluxes (material and energy), thermodynamic forces, multiple steady states, nonzero steady-state flux, and may be part of coupled transport and rate processes $[21,31,36,40,41,79,82,97]$. There is also a pos- itive non-zero entropy production rate, which characterizes the nonequilibrium steady state (NESS). Recent developments in the area of fluctuation theorems have highlighted the importance of entropy production ('dissipation cost') and its relationship with the irreversible nature of a system $[83,98]$ and the robustness of biochemical cycles $[14,42,83,99,100,101]$. Some of the deterministic and stochastic approaches used in the modeling are briefly discussed within the next sections.

\subsection{Deterministic models}

Schlögl's model [100] has an autocatalytic and trimolecular reaction:

$\mathrm{A}+2 \mathrm{X} \underset{k_{1 b}}{\stackrel{k_{1} f}{\rightleftarrows}} 3 \mathrm{X}, \quad \mathrm{B} \underset{k_{2 b}}{\stackrel{k_{2} f}{\rightleftarrows}} \mathrm{X}$.

The concentrations of substrates $C_{A}$ and $C_{B}$ are kept constant with exchange of materials between two material baths. Here $C_{X}$ is the dynamic concentration, $k_{i f}$ and $k_{i b}$ are the forward and backward rate constants for reaction $i$, respectively, and the system is assumed to be homogeneous in space and the volume, The deterministic model $[19,38,102]$ is based on the law of mass action and yields a nonlinear ordinary differential equation given by

$\frac{d C_{X}}{d t}=\left(J_{1 f}+J_{2 f}\right)-\left(J_{1 b}+J_{2 b}\right)$,

where

$J_{1 f}=k_{1 f} C_{A} C_{X}^{2}, J_{1 b}=k_{1 b} C_{X}^{3}, J_{2 f}=k_{2 f} C_{A}, J_{2 b}=k_{2 b} C_{X}$

Here $J_{i f}$ and $J_{i b}$ are the forward and backward reaction fluxes, respectively. Chemical detailed balance (or equilibrium steady state) occurs when $J_{i f}=J_{i b}$ in every reaction, while mathematical detailed balance assumes that total forward rate is equal to total backward rate. As the Schlögl reaction system is cubic (from the trimolecular reaction) there may be three states depending on the set of parameters, such as a bistable state with two stable steady states separated by an unstable steady state. The bifurcation point is obtained through the following discriminant equation [38]

$\Delta=4 k_{1 f}^{3} C_{A}^{3} k_{2 f} C_{B}-k_{1 f}^{2} C_{A}^{2} k_{2 b}^{2}+4 k_{1 b} k_{2 b}^{3}-18 k_{1 f} k_{1 b} C_{A} k_{2 f} k_{2 b} C_{B}+27 k_{1 b}^{2} k_{2 f}^{2} C_{B}^{2}$

The bistable state occurs when $\Delta<0$ [38]. There exist many biological examples of bistability and switching behavior $[38,96,102]$. When the volume $V$ and number of molecules change, the values of $k_{i}$ and $C_{A}$ and $C_{B}$ are assumed to be constant. The steady state equilibrium concentration of dynamic molecule $\mathrm{X}$ is

$$
C_{X, e s}=k_{1 f} C_{A} / k_{1 b}=k_{2 f} C_{B} / k_{2 b} .
$$

"Macroscopic" studies of living cell biochemistry are usually based on deterministic nonlinear differential equations according to the law of mass action [54]. Currently, it is generally accepted that a bistability in the deterministic dynamics corresponds to a bimodal probability density function in the stochastic approach [38]. With the increasing size of chemical reaction systems, there is a separation of time scale: the transition rates between the two macroscopic states become infinitesimal. The driving forces are

$$
\begin{aligned}
& \Delta \mathrm{m}=k_{B} T \ln \left(\frac{J_{1 f}}{J_{1 b}}\right), \\
& \Delta \mathrm{m}_{2}=k_{B} T \ln \left(\frac{J_{2 f}}{J_{2 b}}\right),
\end{aligned}
$$


Table 1

Variety of spatial and time scales of biological system. Nonequilibrium thermodynamics studies complex heterogeneous chemical and biological systems, at all levels of hierarchy, from molecular scale to macromolecular sacle. A "hierarchical system" in the long temporal scheme is open to the exchange of matter, energy, and information with the environment [56-59].

\begin{tabular}{|c|c|c|c|}
\hline Biological systems & $\begin{array}{l}\text { Time } \\
\text { scale }\end{array}$ & $\begin{array}{l}\text { Spatial } \\
\text { scale }\end{array}$ & Modeling and methods \\
\hline $\begin{array}{l}\text { Atoms, molecules: } \\
\mathrm{H}, \mathrm{C}, \mathrm{N}, \mathrm{O}, \mathrm{S}, \ldots\end{array}$ & $\sim \mathrm{fs}$ & $0.1 \mathrm{~nm}$ & Molecular or microscopic scale: \\
\hline $\mathrm{H}_{2} \mathrm{O}, \mathrm{CO}_{2^{\prime}}, \mathrm{N}_{2^{\prime}}$. & & & -Mutations, gene expressions, \\
\hline $\begin{array}{l}\text { Small molecules ( } ~ 30 \text { or fewer } C \text { atoms), Building blocks with } \\
\text { chirality, isomerism, hydrophobicity: } \\
\text { Nucleotides, amino acids, sugars, fatty acids, ... }\end{array}$ & $\sim$ ps-ns & $\sim 1 \mathrm{~nm}$ & $\begin{array}{l}\text {-Signaling, } \\
\text {-Metabolic pathways, } \\
\text {-Cell cycle. }\end{array}$ \\
\hline $\begin{array}{l}\text { Biomolecules (Macromolecules) with collective properties, } \\
\text { conformational transitions: } \\
\text { DNA, proteins, polysaccharides, lipids }\end{array}$ & $\sim \mathrm{ns}$ & $\sim 10 \mathrm{~nm}$ & $\begin{array}{l}\text { Molecular dynamics } \\
\text { reaction kinetics, lattice reaction-annihilation } \\
\text { processes, } \\
\text { stochastic dynamics. }\end{array}$ \\
\hline $\begin{array}{l}\text { Supramolecular structures with self-organization and molecular } \\
\text { recognition: } \\
\text { Enzymatic complexes, membranes, contractile systems }\end{array}$ & & & \\
\hline $\begin{array}{l}\text { Organelles with self-organization, molecules in compartments, } \\
\text { reaction networks, biological cycles: }\end{array}$ & $\sim 10 \mathrm{~ns}$ & $\sim 1 \mu \mathrm{m}$ & Mesoscopic scale \\
\hline $\begin{array}{l}\text { Nucleus, mitochondrion }(1 \mu \mathrm{m}) \text {, } \\
\text { chloroplast }(5 \mu \mathrm{m}) \\
\text { Cells with self-organization, autonomy, self-replication, } \\
\text { metabolism, evolution }\end{array}$ & $\sim 1 \mathrm{~ms}$ & $\sim 100 \mu \mathrm{m}$ & $\begin{array}{l}\text { Cell to cell and cell to matrix interactions, } \\
\text { translocation of a biomolecule } \\
\text { growth phenomena. }\end{array}$ \\
\hline Prokaryotic cell $(0.5-4 \mu \mathrm{m})$ & & & General kinetic equations of Fokker-Planck type, \\
\hline Plant cell $(10-100 \mu \mathrm{m})$ & & & $\begin{array}{l}\text { stochastic dynamics } \\
\text { s. }\end{array}$ \\
\hline Tissues with multicellularity, differentiation, collective behavior & $\sim \mathrm{S}$ & $\sim 1 \mathrm{~mm}$ & $\begin{array}{l}\text { kinetic Monte Carlo. } \\
\text { Macroscopic scale }\end{array}$ \\
\hline $\begin{array}{l}\text { Organs with physiology, information processing, memory, } \\
\text { recognition: } \\
\text { Brain, lungs, liver }\end{array}$ & $\sim 100 \mathrm{~s}$ & $\sim 100 \mathrm{~mm}$ & $\begin{array}{l}\text { Diffusion of nutrients, } \\
\text { cell migration, } \\
\text { tissue processes. }\end{array}$ \\
\hline Organisms with language, sex, social behavior & & $\sim 1 \mathrm{~m}$ & $\begin{array}{l}\text { Reaction-diffusion systems, } \\
\text { continuous mechanics, } \\
\text { finite elements, } \\
\text { finite differences. }\end{array}$ \\
\hline
\end{tabular}

where $k_{B}$ is the Boltzmann constant, $T$ is the temperature. For one forward cycle of reactions the potential energy required to transform one $\mathrm{A}$ into one $\mathrm{B}$ is

$$
\Delta G=\Delta \mathrm{m}_{A B}=k_{B} T \ln \left(\frac{k_{1 f} k_{2 b} C_{A}}{k_{1 b} k_{2 f} C_{B}}\right) .
$$

Therefore, the rate of entropy production in the $i$ th steady state (is) becomes

$\Phi_{i}=\Delta G J_{i s}=k_{B} T\left[\ln \left(\frac{J_{1 f}}{J_{1 b}}\right)\left(J_{1 f}-J_{1 b}\right)+\ln \left(\frac{J_{2 b}}{J_{2 f}}\right) \underset{\left(J_{2 b}-J_{2 f}\right)}{(6)}\right]_{s} \geq 0^{\prime}$

where the steady state flux is

$J_{i s}=\left(J_{1 f}-J_{1 b}\right)_{S}=\left(J_{2 b}-J_{2 f}\right)_{S}$.

\subsection{Stochastic models}

The stochastic model, in the form of the chemical master equation (CME), is an infinite system of mathematically coupled ODEs $[16,38,43,95,103,104]$. Assuming that $n_{a}$ and $n_{b}$ are the number of substrate molecules, which are fixed for a fixed volume, and $P_{n}(t)$ is the probability of having $n X$ molecule at time $t$. The stochastic model equations are

$$
\frac{d P_{0}(t)}{d t}=\mathrm{a}_{1} P_{1}-\mathrm{b}_{0} P_{0}
$$

$$
\begin{aligned}
& \frac{d P_{n}(t)}{d t}=\mathrm{a}_{n+1} P_{n+1}+\mathrm{b}_{n-1} P_{n-1}-\left(\mathrm{a}_{n}+\mathrm{b}_{n}\right) P_{n} \quad n=1, . ., \infty \\
& \text { where } \\
& \mathrm{b}_{n}=\hat{k}_{2 f} n_{b}+\hat{k}_{1 f} n_{a} n(n-1), \mathrm{a}_{n}=\hat{k}_{2 b} n+\hat{k}_{1 b} n(n-1)(n-2),
\end{aligned}
$$

where $\beta_{n}$ and $a_{n}$ are the birth and death rates, respectively. The rate constants $\hat{k}_{i}$ are related to the number of reactants involved in the $i$ th reaction; for example, for a reaction involving $m$ reactants, we have $\hat{k}_{i}=k_{i} / V^{m-1}$. Steady state probability distribution would be obtained by the mathematical detailed balance equation

$\mathrm{b}_{n-1} P_{n-1, s}=\mathrm{a}_{n} P_{n, s}$ where the probability fluxes in forward and backward reactions are equal at each state $[38,102,103]$. So the stationary probabilities become

$$
P_{n, s}=P_{0, s} \prod_{i=0}^{n-1} \frac{\mathrm{b}_{i}}{\mathrm{a}_{i+1}}, \quad P_{0, s}=1-\sum_{j=1}^{\infty} P_{j, s} .
$$

In the CME, the steady-state probability distribution of the equilibrium steady state is a Poisson distribution [104]. For Schlögl's model [81] steady-state probability distributions become 
Table 2

Dynamically coupled processes may lead to systems that acquire emergent properties that cannot be expected from individual processes. Such systems are self-organized systems as they can lower the overall rate of entropy production by dissipating energy $[6,10,18,20,21,22,23,35,37,80,48]$. Self-organization in biological systems is related to a function through the dissipation of ATP [23]

\begin{tabular}{|c|c|c|}
\hline Principles & Mechanisms \& Processes & Biological examples \\
\hline $\begin{array}{l}\text { Nonequilibrium } \\
\text { thermodynamics. }\end{array}$ & $\begin{array}{l}\text { Thermal, chemical or other energy dissipation due to entropy } \\
\text { production in biochemical cycles. }\end{array}$ & $\begin{array}{l}\text { Oxidative phosphorylation; } \\
\text { Hydrolysis of ATP for active transport in living } \\
\text { cells }[21,31,32,41-49,79,133,134] \text {. }\end{array}$ \\
\hline Thermodynamic coupling. & $\begin{array}{l}\text { Emergence of driving }(J X>>0) \text { and driven }(J X<0) \text { processes; } \\
\text { driving process is a spontaneous process, while driven process } \\
\text { needs energy from outside. Here } J \text { and } X \text { show the flow and force, } \\
\text { respectively. }\end{array}$ & $\begin{array}{l}\text { Respiration drives the oxidative } \\
\text { phosphorylation to produce ATP, } \\
\text { ATP hydrolysis drives molecular pumps } \\
{[41,45,81,82] \text {. }}\end{array}$ \\
\hline $\begin{array}{l}\text { Emergence of a new } \\
\text { property, state, flow. }\end{array}$ & $\begin{array}{l}\text { Thermodynamic coupling and collective effects lead to new state } \\
\text { and system properties. }\end{array}$ & $\begin{array}{l}\text { Functional biological cycles; enzymatic } \\
\text { oscillators, information processing }[18,48] \text {. }\end{array}$ \\
\hline $\begin{array}{l}\text { Robustness in selecting and } \\
\text { remaining in a state: the } \\
\text { ability to perform in } \\
\text { uncertainty. }\end{array}$ & $\begin{array}{l}\text { Information processing, feedback loops, physical and chemical } \\
\text { constraints and synchronization between biological cycles, key } \\
\text { properties of biochemical cycles. }\end{array}$ & $\begin{array}{l}\text { Cell-cell regulation and signal transduction } \\
\text {,Transitions between two key states in } \\
\text { biochemical cycles, such as } \mathrm{F}_{\mathrm{o}} \mathrm{F}_{1}-\mathrm{ATPase} \\
\text { Bioenergetics, mutations }[32,70,74,83-86]\end{array}$ \\
\hline $\begin{array}{l}\text { Bifurcation from one steady } \\
\text { state to another. }\end{array}$ & $\begin{array}{l}\text { Distance from global equilibrium, local fluctuations around } \\
\text { critical controlling parameters, nonlinearities, lipid and protein } \\
\text { kinase signals, protein-protein interactions. }\end{array}$ & $\begin{array}{l}\text { Switches between coupled biochemical cycles, } \\
\text { cycles regulatory processes, cell size control with } \\
\text { bursting oscillations }[12,18,32,40,87-91] \text {. }\end{array}$ \\
\hline $\begin{array}{l}\text { Symmetry breaking } \\
\text { by switching from one } \\
\text { symmetry level to another. }\end{array}$ & $\begin{array}{l}\text { Thermodynamic gradients, local fluctuations, homochirality in } \\
\text { biomolecules by breaking racemization. }\end{array}$ & $\begin{array}{l}\text { Nonlinear reactions, Turing patterns, biosystems } \\
\text { produce only D-forms of sugars and L-forms } \\
\text { of amino acids; L-form rotate polarized light } \\
\text { anticlockwise [92]. }\end{array}$ \\
\hline
\end{tabular}

$P_{n, s}=P_{0, s} \frac{\mathrm{q}^{n}}{n !} e^{-\mathrm{q}}, \quad \mathrm{q}=\frac{C_{A} k_{1 f} V}{k_{1 b}}$

When $k_{1 f} k_{2 b} C_{A}=k_{1 b} k_{2 f} C_{B}$ is not satisfied, the system will reach nonequilibrium steady state, in which $J_{i f} \neq J_{i b^{\prime}}$ and the reaction will be forming a cycle, such as $\mathrm{A} \rightarrow \mathrm{X} \rightarrow \mathrm{B} \rightarrow \mathrm{A}$, which may be quantified by a cycle flux $[38,102]$. In biochemical cycles, the concentrations of the substrates could vary in different situations, while the rate constants, which depend on the type of substrates involved, would not change. Biological systems are capable of reaching a unique fluctuating, stochastic, and nonequilibrium stationary state (NESS) with a stationary probability distribution $[40,42,102,103]$. A certain amount of chemical energy is lost to the solvent via heat, which also fluctuates $[38,99]$. There is a mean stationary entropy production rate $\Phi$, which is calculated first over each possible state and later over each reaction [43] using either cycle flux

$$
\Phi_{C M E}=\Delta G \sum_{n=0}^{\infty}\left[\hat{J}_{1 f}(n)-\hat{J}_{1 b}(n+1)\right]=\Delta G \sum_{n=0}^{\infty}\left(\mathrm{b}_{1 n} P_{n, s}-\mathrm{a}_{1 n+1} P_{n+1, s}\right)^{\prime}
$$

where the stochastic forward and backward steady-state fluxes of the reactions are:

$$
\begin{aligned}
& \hat{J}_{1 f}(n)=\mathrm{b}_{1 n} P_{n, s}, \quad \hat{J}_{1 b}(n)=\mathrm{a}_{1 n} P_{n, s}, \\
& \hat{J}_{2 f f}(n)=\mathrm{b}_{2 n} P_{n, s}, \\
& \text { and separation of birth and death rates from each reaction yields }
\end{aligned}
$$

$$
\begin{aligned}
& \mathrm{b}_{1 n}=k_{1 f} C_{A} n(n-1) / V, \quad \mathrm{~b}_{2 n}=k_{2 f} C_{B} V, \\
& \mathrm{a}_{1 n}=k_{1 b} n(n-1)(n-2) / V^{2}, \quad \mathrm{a}_{2 n}=k_{2 b} n,
\end{aligned}
$$

with

$$
\mathrm{b}_{n}=\mathrm{b}_{1 n}+\mathrm{b}_{2 n}, \quad \mathrm{a}_{n}=\mathrm{a}_{1 n}+\mathrm{a}_{2 n} \text {. }
$$

When the system is bistable, there are multiple entropy production rates one for each steady state and the stochastic entropy production rate becomes a combination of the rates in each possible state, weighted by the probability of being in each of those states. This shows that the entropy production rate is neither maximized nor minimized for all NESSs, and hence the entropy production rate alone may be unable to fully describe stability in the steady state [64,105]. However, as Han and Wang [99] have suggested lower rate of entropy production indicates less dissipation in a complex biochemical network, which may be more robust to perturbations.

The deterministic and the stochastic models under equilibrium conditions are in agreement; when there is only one real root, steady-state behavior of the stochastic system will be the same with that of deterministic system. However, when the system is bistable, the behavior in the deterministic model depends on the initial condition, while the steady-state behavior in the stochastic model is independent of the initial condition [38]. In the long term, the stochastic model predicts that the system spends almost all its time in the two FCA states that correspond to the stable states of the deterministic model, and the proportion of time spent in each is dictated by the ratio of the transition rates between them. The key behavior of a bistable system is the ability of transition between the FCAs. Schlögl's model exhibits multiple time scales: at fast scale the system relaxes to one of the FCAs, while it transitions from one to another FCA at a slow scale $[38,88,100]$.

Modeling and analysis of biochemical systems need consideration of multiple scales, which require linking and integrating the models that operate at different temporal and spatial scales. To improve multiscale modeling of data, new and existing strategies for coupling micro- and macro level models must be developed and tested $[13,29,96]$. Vellela and Qian [38] have suggested a new hybrid method with continuous diffusion and discrete jumps, which may describe the multiscale dynamics of bistable systems [96]. For a large but finite volume size, the stochastic model predicts that bistable systems will be in the more stable FCA and spends most of its time there. However, for small volume, the FCA may exchange 


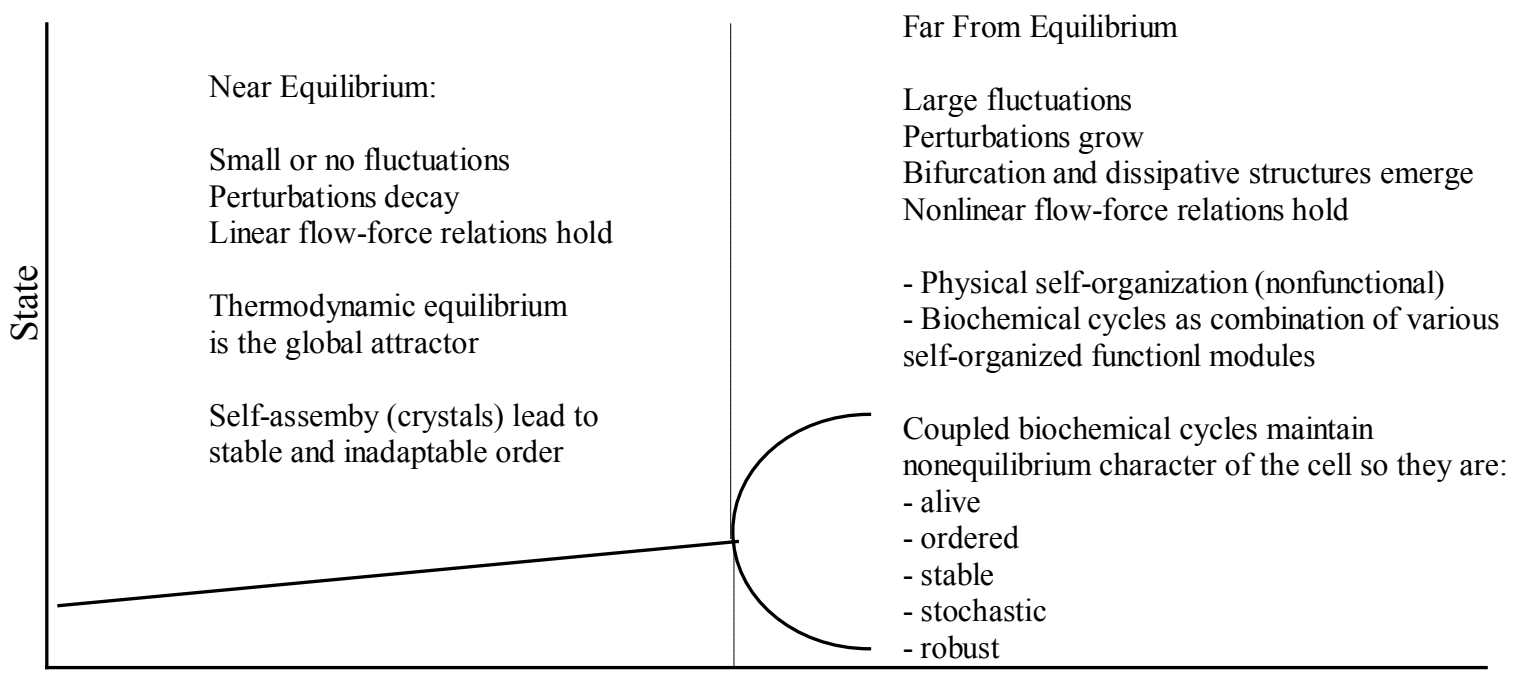

$\mathrm{Xc}$

\section{Distance From Equilibrium}

Figure 1. Thermodynamic branch [48] showing the properties of physical and biological systems away from global equilibrium. Far from global equilibrium emerges after a critical distance from equilibrium characterized by critical thermodynamic force Xc. Nonequilibrium thermodynamics leads to the conclusion that in systems moved away from equilibrium, self-organized dissipative processes can emerge with an ability to reduce the effect of the applied gradient. As biological systems increase their total dissipation, they can develop more complex structures with more matter, energy, and information flow, increase their cycling activity, develop greater diversity, and generate more hierarchical levels. Nonequilibrium" or "out of equilibrium" becomes incomplete, arbitrary, and misleading unless it is clearly specified as 'near equilibrium' or 'far from equilibrium' $[30,43,46,78]$. In the vicinity of global equilibrium only, linear phenomenological equations between

flows $(J)$ and forces $(X)$ exist $\left(J_{i}=L_{i k} X_{k}\right.$ ) and the cross coefficients obey Onsger's reciprocal rules. However, for systems far from global equilibrium and near

equilibrium the rate of entropy production is the product of independent flows and forces ( $\Phi=\sum_{i} J_{j} X_{i}$ ).Linear flow-force relations are valid when the Gibbs free energy ranges less than $1.5 \mathrm{~kJ} / \mathrm{mol}$ for chemical reactions $[31,32,48,49]$. The formalism of LNET can be used in wider ranges (over a $7 \mathrm{~kJ}^{-1}$ ) than usually expected with an error in the reaction velocity less than $15 \%[28,31,45-49]$ for some reactions and for selected biological pathways.

relative stability with the changes solely in volume [38]. The longterm behavior of bistable systems is determined by the transition rates between the FCAs. The CME model is the most valid mathematical model to describe nonequilibrium systems occurring at the miscroscopic and mesoscopic scales [38,104]. The mesoscopic theory of stochastic macromolecular mechanics and macroscopic nonequilibrium thermodynamics are consistent, which can have a wide range of applications to cellular and molecular biology. However, a proper mathematical framework would be based on stochastic differential equations, which may be explicit and discrete with master equation type or continuous in time and state space with the FokkerPlanck equation.

\subsection{Multiscale computer simulations}

Computer simulations explore an agreement between a model and experimental data. Some special challenges associated with biochemical simulations are: (1) biochemical systems involve combinatorially many chemical compounds and reaction systems, (2) kinetic parameters for biochemical cycles are rarely known, (3) molecular species have diverse dynamic time scales, and (4) modeled systems lack of a comprehensive interpretation power of identifying vital connections between its microscopic and macroscopic properties $[17,57]$. Such an interpretation can be aided by coarse-graining, in which certain nodes and/or reaction processes are merged and/or eliminated [106] or group of atoms are combined into systems of rigid bodies connected by joints [107]. Ideally, one wants to substitute multiple elementary (single-step, Poisson-distributed) biochemical reactions with a few complex processes in a way that retains predictability of the system. This may be possible by reducing the number of interacting elements, increasing the copy numbers of agglomerated hyper species, and combining multiple microscopic rates into a smaller number of effective parameters $[28,30,57,65]$. Multiscale approaches possess an inherent uncertainty that needs to be addressed by setting standards for data analysis. For example, uncertainty can be controlled by using numerical simulation techniques to approximate with quantitative error indicators on different scales leading to multiscale numerical simulation environments. Still, understanding which measurements are most informative for characterizing a complex biological system will require significant advances in computational modeling. The disparity between the current scale of biological modeling and analytical technologies is one of the most severe bottlenecks in systems biology. There is unlikely to be a single "best" approach for modeling complex biological systems. Instead, the most effective methodologies probably will link analytical measurements and data analysis to models of a particular biological response. Building a predictive model requires the ability to measure effects and compare them with the simulated results $[17,39,58,106]$.

A well known example of coarse graining in biochemisty is the Michaelis-Menten (MM) kinetics

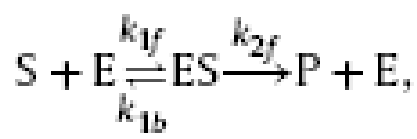


where $k_{1 \rho} k_{1 b}, k_{2 f}$ are the kinetic rates, S, ES, E, and P are the substrate, enzyme-substrate complex, enzyme, and product, respectively. Here, the enzyme catalyzes the $\mathrm{S} \rightarrow \mathrm{P}$ transformation by combining with $S$ to create a complex ES, which dissociates either back to E+S or forward to E+P. When $S>>E$, the enzyme cycles many times, and in a coarse-grained reaction of $\mathrm{S} \stackrel{J_{r}}{\longrightarrow} \mathrm{P}$ we have the rate of reaction cycle given by $J_{r}=k_{2 f}[\mathrm{~S}][\mathrm{E}] /\left\{[\mathrm{S}]+\left(k_{2 f}+k_{1 b}\right) k_{1 f}\right\}$.

However, the coarse-grained reaction of Eq. (15) is insufficient when stochasticity is important as each MM reaction consists of multiple elementary steps, thus approximating the number of the reactions as a Poisson variable is not always valid [65]. A coarse-graining procedure must be applicable to systems with variable time scales by eliminating the time scales hierarchically [106]. The reducibility of complex system by coarse graining may determine how to model it (i.e., microscopically or macroscopically) and at which scale to measure it $[19,26,65]$. Still, where possible, one should chose not to excessively coarse-grain the state space if it will reduce the precision or predictive power of the model [106]. In coarse-graining as well as in relating single-molecule dynamic disorder to reaction networks, one must understand if the fluctuations are enhanced or damped [12]. The nature of fluctuations is closely related to the distance from equilibrium as the thermodynamic branch shown in Figure 1 illustrates.

Enzyme kinetics in complex biochemical cycles [19] may also involve the effects of inhibitors and activators of the enzyme. Crampin et al. [19] generalized the enzyme inhibition by the following scheme

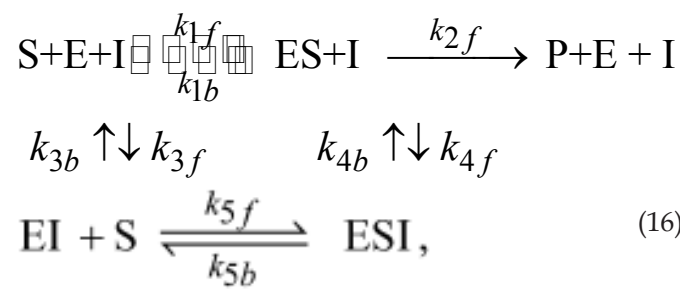

where I is an inhibitor and EI and ESI are enzyme-inhibitor complex and enzyme-substrate-inhibitor complex, respectively. For the inhibitor and substrate inhibitor equilibrium constants are $K_{I}=k_{3} / k_{3 b}$ and $K_{S I}=k_{4} / k_{4 b}$, respectively, and the inhibition is noncompetive if $K_{t}$ $=K_{S I}$. The MM type of kinetics exhibits saturation at high substrate concentration, which is another common behavior of enzymatic reactions [19].

Extreme pathways correspond to steady-state flux distributions through a metabolic network and help characterize the relative flux levels through all the reactions necessary to convert substrates to products, balance all cofactor pools, and study emergent properties $[75,108]$. The minimal extreme pathway length indicates the minimum number of reactions necessary to synthesize a given product, including a set of variable reactions in addition to the essential reaction set. For example, the number of essential reactions in aspartic acid production in Helicobacter pylori and Haemophilus influenza are 12 and 22, respectively, while the utilized reactions are 91 and 97 , respectively $[68,108]$. Here the essential reactions are the number of reactions used in every extreme pathway, while the utilized reactions are used at least once in the set of extreme pathways for the production of the associated product. The determination of these essential reaction sets could enable the identification these reactions that would result in the complete failure of the synthesis capability for the corresponding product. It will also display a certain degree of redundancy in the network and an ability of the metabolic network to make a selection in how the product is synthesized.

Ge and Qian [102] analyzed the phosphorylation-dephosphory- lation biochemical cycle $[79,109]$, which is known as a cellular biochemical signaling module, [95] using Ferrel's kinetic model [88]

$\mathrm{ATP}+\mathrm{E}+\mathrm{K}^{*} \underset{k_{1 b}}{\stackrel{k_{1}}{\rightleftarrows}} \mathrm{E}^{*}+\mathrm{ADP}+\mathrm{K}^{*}, \quad \mathrm{E}^{*}+\mathrm{P} \underset{k_{2 b}}{\stackrel{k_{2}}{\rightleftarrows}} \mathrm{E}+\mathrm{P}_{\mathrm{i}}+\mathrm{P}, \quad \mathrm{K}+2 \mathrm{E}^{*} \underset{k_{3 b}}{\stackrel{k_{3}}{\leftrightarrows}} \mathrm{K}^{* \prime}$

where $\mathrm{E}$ and $\mathrm{E}^{*}$ are active and inactive forms of a signaling protein, $\mathrm{K}$ and $\mathrm{P}$ are kinase and phosphatase enzymes that catalyze the phosphorylation-dephosphorylation, and $\mathrm{K}$ and $\mathrm{K}^{*}$ active and inactive forms of the kinase. This cycle is bistable in a certain parameter range [109]. In a cell, concentrations of $\mathrm{K}, \mathrm{P}, \mathrm{ATP}, \mathrm{ADP}$, and $\mathrm{P}_{\mathrm{i}}$ are constant, and the free energy

$$
\Delta G=k_{B} T \ln \left(k_{1 f} k_{2 f}[\mathrm{ATP}] /\left\{k_{1 b} k_{2 b}[\mathrm{ADP}]\left[\mathrm{P}_{\mathrm{i}}\right]\right\}\right)
$$

comes from the hydrolysis of ATP $\left(\mathrm{ATP}+\mathrm{H}_{2} \mathrm{O} \square \mathrm{ADP}+\mathrm{P}_{\mathrm{i}}\right.$ ) where ADP is adenosine diphosphate, and $\mathrm{P}_{\mathrm{i}}$ is phosphate). Thermodynamic limit of a bistable phosphorylation-dephosphorylation cycle plays role in the selection of more stable state, and the relative stability and robustness of the cycle cannot be analyzed without an explicit consideration of the intrinsic fluctuations in the model as the minor perturbations are inevitable in living systems $[14,38,99,102]$. Huang and Qian [109] also analyzed the phosphorylation-dephosphorylation cycle using a simple MM kinetics, which shows that depending on the level of substrate concentration [S], the catalyzed reaction can be either first order when the Michaelis constant $K_{\mathrm{M}}>>$ [S] or zeroth order when $K_{\mathrm{M}}<<$ [S]. Dynamics of the system exhibits the canonical competition behavior beside the bistability [109-111]. Beard et al. [71] analyzed the energy metabolism network (glycolysis, tricarboxilic acid cycle, oxidative phosphorylation, and ATP hydrolysis) with 28 reactions [112]. The CME is a fundamental mathematical theory for mesoscopic biochemical reaction systems in a small, spatially homogeneous volume [95], and its large volume limit recovers the law of mass action. The deterministic differential equations, while defining various attractors, provide no information on the relative probabilities between them. The Maxwell-type construction incorporated in the CME leads to a more complete theory [102].

\subsection{Network dynamics}

Consider an $n$ component network. The $n$ components may be the relevant numbers of metabolites in a metabolic network or the relevant proteins in a gene regulatory network pathway $[11,30,67,68,113]$ or other quantities specifying the network. The $n$-dimensional transpose vector $\mathbf{q}^{T}=\left(q_{1}, q_{2}, . ., q_{n}\right)$ is the state variable of the network and the value of $j$ th component is denoted by $q_{j}$. Let $f_{i}(\mathbf{q})$ be the deterministic non-linear force on the $j$ th component, which includes both the effects from other components and itself, and $\zeta_{j}(\mathbf{q}, t)$ the random force. For simplicity, assume that $f_{i}$ is a smooth function explicitly independent of time. The network dynamics may be generally modeled by a set of standard stochastic differential equations assuming that the noise will be Gaussian and white $[5,11,15]$

$\frac{d q_{j}}{d t}=f_{j}(\mathbf{q})+\mathbf{z}_{j}(\mathbf{q}, t)$.

In complex biochemical network more complicated noises, such as non-Gaussian and colored, can exist. If an average over the stochastic force $\zeta$ is performed, Eq. (18) is reduced to the deterministic equation in dynamical systems. Eq. (18) does not address how the stochastic force $\zeta$ can be related to the deterministic force $f$. To do that Eq. (18) can be transformed into the following form [11]:

$[S(\mathbf{q})+T(\mathbf{q})] \frac{d \mathbf{q}}{d t}=-\nabla \mathbf{f}(\mathbf{q})+\mathbf{z}(\mathbf{q}, t)$, 
ric matrix $T$, and the single-valued scalar function $\phi$. The symmetric matrix term is 'degradation' $\dot{\mathbf{q}}^{T} S(\mathbf{q}) \dot{\mathbf{q}} \geq 0$ [11,15], while the antisymmetric part is non-decaying: $\dot{\mathbf{q}}^{T} T(\mathbf{q}) \dot{\mathbf{q}}=0$. The degradation is represented by the symmetric matrix $S$ (the friction matrix) and the transverse force by the antisymmetric matrix $T$ (mass, magnetic fields, rotation). Ao [11] introduced $\phi(\mathbf{q})$ as an emerging property of the network and called the network potential with respect to the network state variable q. It is not the usual free energy, although it plays the same role as energy function in thermodynamics. The potential $\phi(\mathbf{q})$ describes what the network eventually like to be under all thermodynamic and other constraints, and determines the robustness of network dynamics. Consequently, the tendency for optimization of network requires the minimum of potential $\phi(\mathbf{q})$ and without the stochastic effect in Eq. (18), no unique potential function can be determined $[11,15]$. Eq. (19) may identify the dynamical structure and would be suitable for analyzing metabolic networks directly.

In order to have a unique identification, Ao [11] has imposed a constraint on the stochastic force so the symmetric matrix in Eq. (19) to be semi-positive definite

$$
\left.\left\langle\mathbf{z}_{j}(\mathbf{q}, t) \mathbf{z}^{T}\left(\mathbf{q}, t^{\prime}\right)\right\rangle=2 S(\mathbf{q}) \mathrm{d}\left(t-t^{\prime}\right) \text { and } \underset{(20)}{\langle\mathbf{z}}(\mathbf{q}, t)\right\rangle=0
$$

where $\delta(t)$ is the Dirac delta function. This constraint is consistent with the Gaussian and white noise assumption for $\zeta$ in Eq. (18). The matrix $[S+T]$ at left hand side of Eq. (19) makes the network tend to the minimum of potential function $\phi(\mathbf{q})$, leading to an optimization. The stationary distribution function $P_{0}(\mathbf{q})$ for the state variable is a type

of Boltzmann-Gibbs distribution: $P_{0}=(1 / Z) \exp [-f(\mathbf{q})]$ with the partition function $Z$ given by $Z=\int d^{n} \mathbf{q} \exp [-\mathbf{f}(\mathbf{q})]$ . This allows a direct comparison with the stochastic experimental data at steady states. The present stochastic modeling, Eq. (19), indicates two time scales: the very short one characterizing the stochastic force $\zeta(\mathbf{q}, t)$ and the time scale on which the smooth functions of $\phi(\mathbf{q})$, degradation (friction) matrix $S(\mathbf{q})$ and the translocation matrix $T(\mathbf{q})$ are well defined. This corresponds to the hierarchical structure of metabolic pathways [11,68-74,112].

The stochastic force $\zeta(q, t)$ can arise from the environmental influence on the network, or from approximations such that the continuous representation of a discrete process. The friction matrix $S(\mathbf{q})$ and the frictional force $S$ show that the network has the tendency to approach to a steady state. The present dynamical structure theory requires that the friction is always associated with the noise according to the relation in Eq. (20). The friction and noise are the two opposite sides of stochastic dynamics that are the ability to adaptation with friction and the ability to optimization with noise [11,30,71-74].

\subsection{Molecular motors}

Many single biochemical molecules of large proteins, such as enzymes (ranging from 2-100 $\mathrm{nm}$ ), are mainly characterized by length scales in the nanometer-to-micrometer range and dissipation rates of 10-1000 $k_{B} T$ per second $[29,114]$. A single macro molecule with $n$ modification sites can exist in $2^{n}$ microscopic key states [25,54]. Motor proteins utilize biochemical energy to perform mechanical work. Recent advancements in single-molecule experimental techniques and the results of structural genomic projects may be very helpful in understanding protein functions and enzyme kinetics on the molecular scale $[29,83,114-121]$. However, understanding protein functions requires knowledge and consideration of their multiprotein complex or metabolic network. This needs bridging many orders of magnitude in spatial and temporal dimensions [13,29,30,39,56,57]. Enzymes are highly specific and efficient in their acceleration of biochemical reaction rates by orders of magnitude. As Baker [117] not- ed, under physiological conditions, the ATP hydrolysis reaction is energetically favorable but slow; however, enzyme catalysts dramatically accelerate the rate of hydrolysis and harness the energy to repeat a cyclical sequence of catalytic events capable of carrying out useful functions. The free energy from ATP hydrolysis depends on the ratio of $[\mathrm{ATP}] /[\mathrm{ADP}]\left[\mathrm{P}_{\mathrm{i}}\right]$. The amount of energy required for fulfilling a function, the shape and conformational structure (key state) of the enzyme, and the information encoded determine the enzyme dynamics [29]. Biological systems, including molecular motors, in nonequilibrium steady states have net flows and require a continuous input of material, energy, and information to maintain their self-organized steady state as they continuously dissipate net energy $[67,117]$.

Modeling of motor proteins, such as kinesin and myosin-5, $[93,118,121,122]$ must take into account the collective behavior that is the energy coupling between the internal biochemical cycle of a macromolecule and its external load, such as random walk [114,115,118]. Kinetics of the simple model may be characterized by a random walk for motor stepping that is the stochastic movement and a biochemical cycling. The random walk is biased with birth and death processes for an elastic or viscous load [114,119]. These molecular motors are mechanochemical and stochastic systems, and take part in the cellular metabolism under nonequilibrium conditions. As Andrieux and Gaspard [50] states, one of the major achievement would be to understand the nonequilibrium statistical thermodynamics of these motors because of their size, random motion on a privileged direction on average, and dependence of the mean motion on the concentrations of reactants and products.

Molecular motors, over the course of their enzymatic cycle, perform work, as they move along a track a distance $\Delta x$ against a constant force $F$. There are several models for explaining the relationship between a motor's enzymatic mechanisms and its mechanisms for work production $[93,114,117,118]$. In some motor models, enzymatic mechanisms explicitly are different from the work related mechanisms; for example, in the Huxley-Hill model [24] motor force is generated within the biochemical step and work is subsequently performed when a motor relaxes within the potential well of a biochemical state. According to fluctuating thermal ratchet model [120], motor force is generated when a ratchet potential is switched on and work is subsequently performed when a motor relaxes. On the other hand, some recent studies support a chemical motor model $[93,114,121]$ in which reaction and space coordinates are intimately linked. Force is generated and/or work is performed with a thermally activated biochemical transition. For example, a motor structural change induced by ligand binding or by other effects might directly perform work. Most chemical motor models assume that it is the external work $\left(W_{e x t}=F \Delta x\right)$, i.e. in moving the track, that is coupled to the free energy for that step. Internal work, on the other hand may involve pulling out compliant elements in the motor, and is performed in stretching these internal elastic elements that are coupled to free energy $\Delta G$. Motor enzymes, like myosin and kinesin, move along a track while catalyzing a hydrolysis reaction of ATP are selfconsistent mechanochemical systems $[93,117,121]$, in which the reaction mechanisms start and end with free enzyme, while the free enzyme is binded with the substrates and unbinded with products in some random order [122].

For a reaction at isobaric and isothermal conditions, the affinity or the Gibbs free energy difference characterizes the distance from equilibrium. Chemical reactions are usually far from from global equilibrium. The fundamental equation for a chemical reaction sys-

tem operating in a steady state, such as $\mathrm{B} \underset{k_{b}}{\stackrel{k_{f}}{\rightleftarrows}} \mathrm{B}^{\prime}$, is

$\frac{J_{r f}}{J_{r b}}=\exp \left(\frac{A}{R T}\right)$ with 


$$
A=-\sum_{i} \mathrm{n}_{i} \mathrm{~m}, \quad J_{r f}=k_{f}[\mathrm{~B}], \quad J_{r b}=k_{b}\left[\mathrm{~B}^{\prime}\right], \quad J_{r}=J_{r f}-J_{r b}
$$

Here $A$ is the thermodynamic driving force called the affinity, $J_{r f}$ and $J_{r b}$ are the forward and backward reaction rates, respectively, $J_{r}$ is the net reaction rate, and $v_{i}$ is the stoichiometric coefficient, which is positive for product and negative for reactants. Since $J_{r} / J_{r b}+1=\exp (A /$ $R T)$, the net reaction rate or net flow is approximately proportional to the backward reaction rate $J_{r b}: J_{r}=J_{r b}[\exp (A / R T)]$. Eq. (21) provides a relation between affinities, metabolite concentrations, and reaction flows, and is a generalization of the chemical equilibrium condition $A=0$ and $J_{r f}=J_{r b}$, to the case of a chemical system occurring in a nonequilibrium steady state $[69,122]$. Most biochemical reactions, however, involve many simultaneous elementary steps, and the change of concentration of a species would be a sum of rates of change due to those elementary steps in which that species takes place [19].

The reaction rate of a single enzyme molecule fluctuates, which is a general feature of enzymes. A single molecule turnover time that is the time for one enzyme molecule to complete a reaction cycle also fluctuates. As these fluctuations are random, their effect averages to zero over a long period of time or for a large number of molecules, and the MM kinetics well describe some enzymatic reactions [12]. Their stochastic behavior is experimentally observed, and their motion is unidirectional only on average and stops at the thermodynamic equilibrium. Considerable experimental and modeling work exist on the dynamics of linear molecular motors such as actin-myosin or the kinesin-microtubule powered by ATP as well as rotary motors such as $\mathrm{F}_{0} \mathrm{~F}_{1}$-ATPase and bacterial flagellar powered by proton flow across a membrane $[83,118,120]$. The myosin protein is an ATPase and use the chemical energy released by the hydrolysis of ATP to create directed mechanical motion [114]. All the myosin motor proteins share the same biochemical reaction pathway when hydrolyzing ATP [118]. They operate far from equilibrium, dissipate energy continuously, and make transitions between steady states [29]. The enzyme reactions are also coupled with some other processes, such as transport processes characterized by longer time scales. It is also known that the catalytic activity of enzyme molecule is very sensitive to its molecular conformation transitions, which may occur on longer time scales (seconds) compared with time scales for the enzymatic reactions (milliseconds) [12]. The thermodynamic driving force of an enzymatic cycle $\Delta \mu$, can be extracted by the nonequilibrium turnover time traces of single enzyme molecules in living cells that might be measurable experimentally. From chemical master equations under nonequilibrium steady state, the ratio between the probability of $M$ forward turnovers $P\left(d n_{t}=M\right)$ and that of $M$ backward turnovers $P\left(d n_{t}=-M\right)$ is

$$
\frac{P\left(d n_{t}=M\right)}{P\left(d n_{t}=-M\right)}=\exp \left(\frac{\Delta \mathrm{m}}{k_{B} T} M\right)
$$

where $M$ is the positive integer [28,69]. Eq. (22) is the consequence of microscopic reversibility and general as long as the enzyme completes a full cycle, even when the enzyme molecules exhibit more complex kinetic pathways $[14,27,99]$.

In a stochastic description of a transition $\mathrm{B} \underset{k_{b}}{\stackrel{k_{f}}{\rightleftarrows}} \mathrm{B}^{\prime}$

the probability of finding the system in a state of $\mathrm{B}$ at time $t$ is given by the master equation $\frac{d P(B, t)}{d t}=\sum\left(-k_{f} P_{B}+k_{b} P_{B^{\prime}}\right)$

The entropy for a stochastic macromolecular mechanics becomes

$S=-k_{B} \int P(B, t) \ln P(B, t) d B$. In a nonequilibrium stationary states where the probabilities are time independent $\left(d P_{s} / d t=\right.$ $0)$, the entropy production is

$\Phi_{s}=\frac{1}{2 T} \sum\left(J_{r B} A_{B}\right)$,

where $J_{r}$ is the flux and $A$ is the affinity

$J_{r B}=k_{f} P_{B}-k_{b} P_{B^{\prime}}, A_{B}=\ln \left(\frac{k_{f} P_{B}}{k_{b} P_{B^{\prime}}}\right)$. 25)

The $\mathrm{H}$ theorem states that the entropy production is always positive and vanishes when the detailed balance is satisfied

$k_{f} P_{B, e q}=k_{b} P_{B^{\prime}, e q}$.

Movement of the motor protein can be modeled by the Smoluchowski equation $[115,116]$ by assuming that the center of mass of the motor protein as a Brownian motion [120] with the presence of a periodic energy potential

$$
\frac{\partial P(x, t)}{\partial t}=D \frac{\partial^{2} P(x, t)}{\partial x^{2}}-\frac{\partial}{\partial x}\left(\frac{F(x)}{\mathrm{b}} P \underset{(26)}{x}\right),
$$

where $P(x, t)$ is the probability density function of the motor protein at position $x$ and for time $t, D$ and $\beta$ are the diffusion and friction coefficients, respectively. $F(x)$ is the force of the potential and represent the molecular interaction between the motor protein and its track. The driving force for a motor protein comes from the hydrolysis of ATP

$$
\mathrm{ATP}+\mathrm{H}_{2} \mathrm{O} \underset{k_{b}}{\stackrel{k_{f}}{\rightleftarrows}} \mathrm{ADP}+\mathrm{P}_{\mathrm{i}}
$$

This reaction is well characterized by a two-state Markov process (or more generally, $m$ discrete states) [115]

$$
\begin{aligned}
& \frac{d P_{A T P}}{d t}=-k_{f} P_{A T P}+k_{b} P_{A D P}, \\
& \frac{d P_{A D P}}{d t}=k_{f} P_{A T P}-k_{b} P_{A D P} .
\end{aligned}
$$

By introducing internal conformational states to the Brownian particle and to coupling the hydrolysis of ATP with the motor protein movement given in Eq. (26) leads to the following reaction-diffusion system for the movement of a Brownian particle with internal structures and dynamics

$\frac{\partial P(x, n, t)}{\partial t}=D \frac{\partial^{2} P(x, n, t)}{\partial x^{2}}-\frac{\partial}{\partial x}\left(\frac{F(x)}{\mathrm{b}} P(x, n, t)\right)-k_{f n k}(x) P(x, n, t)+k_{b k n}(x) P(x, k, t)$

where $P(x, n, t)$ is the probability of a motor protein with internal state $n$ and external position $x$, and $k_{\text {fnk }}$ is the transition rate constant from internal state $n$ to state $k$ when the protein is located at $x$. The states 
$n$ and $k$, such as attached and detached states, driven by the ATP hydrolysis leads to a biased motion of the motor protein, in which the chemical energy of the hydrolysis of ATP is converted to the mechanical motion of the motor protein [41,114-116]. In general, the stationary solution of Eq. (29) will be a nonequilibrium steady state with positive entropy production and heat generation. Qian [116] considered the possibility of the proteins internal conformational change due to the external work in Eq. (29), while Andrieux and Gaspard [50] extended the analysis to the rotating molecular motors.

\section{Fluctuation theorems}

The fluctuation theorems state that the ratio of the probabilities for forward and backward displacements is related to the rate of entropy production during a given time $[12,28,50,98,101,105]$. These theorems provide a quantitative description of entropy production in finite systems and relate the probability of observing a process of duration $t$ with entropy production $\Phi_{\mathrm{t}}=\sigma$ to that a process with the same magnitude of entropy change, but where the entropy is consumed rather than produced, by $[26,27,114]$

$$
\frac{P\left(\Phi_{t}=\mathrm{s}\right)}{P\left(\Phi_{t}=-\mathrm{s}\right)}=\exp \left(\frac{\mathrm{s}}{R T}\right) .
$$

where the rate of exponential growth is equal on average to the ther-

modynamic entropy production $\sigma=(1 / T) \sum_{i} A_{i} J_{i} \geq 0$. This is known as the Crooks stationary fluctuation theorem [123-124], and establishes that entropy production can be related to the dynamical randomness of the stochastic processes. Therefore, the fluctuation theorem strongly relates the statistics of fluctuations to the nonequilibrium thermodynamics through the entropy production estimations by the second law of thermodynamics. The theory predicts that entropy production will be positive as either the system size or the observation time increases and the probability of observing an entropy production opposite to that dictated by the second law of thermodynamics decreases exponentially. The Crooks fluctuation theorem was used to estimate the free energy difference associated to the unfolding of a RNA molecule [124]. Andrieux and Gaspard [50] also, experimentally proved that the probabilities of the timereversed paths decay faster than the probabilities of the paths themselves and the thermodynamic entropy production arises from the breaking of the time-reversal symmetry of the dynamical randomness. Self-organizing processes of biochemical cycles produce less entropy leading to the higher probability distribution of the opposite values of the fluxes (negative entropy production) and increased capacity for collective behavior and robustness $[48,99]$.

If it is applied to the transient response of a system, the theorem is referred to as the transient fluctuation theorem, which bridges the microscopic and macroscopic domains and links the time-reversible and irreversible descriptions of processes $[26,27,83]$. The transient fluctuation theorem describes how irreversible macroscopic behavior evolves from time-reversible miscroscopic dynamics as either the observation time or the system size increases and how the entropy production can be related to the forward and backward dynamical randomness of the trajectories or paths of systems as characterized by the entropies per unit time $[27,28]$. The new theorems and experimental techniques serve as a basis for the nonequilibrium thermodynamics analyses of small systems, such as mesoscopic systems. Consequently, the mesoscopic theory of stochastic macromolecular mechanics [39] and macroscopic nonequilibrium thermodynamics can have a wide range of applications to cellular and molecular biology $[10,29,31,42]$.

The thermal bath allows macromolecules to exchange energy with the molecules of the solvent through the breakage of weak molecular bonds that trigger the relevant conformational changes. The amount of energies involved in single macromolecules are few tens of $k_{B} T$, small enough for thermal fluctuations over timescales to be relevant in many molecular processes [114]. In thermodynamics of small systems, a control parameter may define the system's state [29,114]; for example, a motor molecule can be described by an internal configuration $\left\{x_{i}\right\}$ and a control parameter $x$ (there can be finite number of control parameters), then $u\left(\left\{x_{i}\right\}, x\right)$ is the internal energy of the system. Upon variation of the control parameter $x$, energy conservation yields

$d u=\sum_{i}\left[\left(\frac{\partial u}{\partial x_{i}}\right)_{x}+\left(\frac{\partial u}{\partial x}\right)_{\left\{x_{i}\right\}} d x\right]=\mathrm{d} Q \underset{(31)}{+\mathrm{d} W}$.

$W=\int_{0}^{x} f F(\{x i\}, x) d x$,

where $x_{f}$ is the perturbation for a time $t_{p}$ and $F\left(\left\{x_{i}\right\}, x\right)$ is the fluctuating force acting on the molecule $F\left(\left\{x_{i}\right\}, x\right)=(\partial u / \partial x)_{\left\{x_{i}\right\}}$.

Since the force is a fluctuating quantity, $W, Q$, and $\Delta u$ will also fluctuate for different trajectories, and the amount of heat or work exchanged with the bath will fluctuate in magnitude and in sign [29]. Therefore, random fluctuations dominate the thermal behavior in small systems. The time evolution of $\left\{x_{i}\right\}$ and therefore the force will change from one perturbation to another, and the system will follow different trajectories. A quantity that characterizes the stochastic nonequilibrium process is the probability distribution of work values $P(W)$ obtained along different trajectories. The average work

over all trajectories $\langle W\rangle=\int W P(W) d W$ is larger than the reversible work and equal to the free-energy difference $\Delta G$ between the equilibrium states defined at $x=x_{f}$ and $x=0$. If we define the dissipated work along a given trajectory as $W_{\text {dis }}=W-\Delta G$, second law can be written as, $W_{d i s} \geq 0$. The equality occurs only when the perturbation process is carried out infinitely slowly in a quasi-static process to relax to equilibrium at each value of the control parameter. On the other hand, nonequilibrium processes are characterized by hysteresis phenomena and the average work performed upon the system differs between a given process and its time-reversed one. Under the assumption of microscopic reversibility (detailed balance), fluctuation theorems assert relations between the entropy production along a given forward process and backward process by $[29,114]$

$$
\frac{P_{f}(W)}{P_{b}(-W)}=\exp \left(\frac{W_{d i s}}{k_{B} T}\right)
$$

where $P_{f}(W)$ and $P_{b}(-W)$ are the work distributions along the forward and backward processes, respectively. Eq. (33) indicates that a steady-state system is more likely to deliver heat to the bath ( $W$ is positive) than it is to absorb an equal quantity of heat from the bath ( $W$ is negative) and hold for any finite time [29,114]. Nonequilibrium steady state systems always dissipate heat on average, therefore, Eqs. (21), (22), (30), and (33) are fundamental relations to describe them.

\subsection{Application to the $\mathrm{F}_{0} \mathrm{~F}_{1}$-ATPase molecular motor}

ATP is synthesized by rotational catalysis in the $\mathrm{F}_{1}$ domain of mitochondrial $\mathrm{F}_{0} \mathrm{~F}_{1}$-ATPase [125-127] shown in Figure 2. The domain $\mathrm{F}_{\mathrm{O}}$ consists of one $a$, two $b$ and a ring of $9-15$ subunits $c$ depending on the species. The subunits $\mathrm{c}$ form a ring, connected to the domain $\mathrm{F}_{1}$ via the subunit $\varepsilon$ and then $\gamma$ and two subunits $b$ and $\delta$. Water-soluble $F_{1}$ domain has the subunits $\alpha_{3} \beta_{3} \gamma \delta \varepsilon$. Catalytic nucleotide-binding sites are formed by each of three subunits $\beta$. The chirality (handedness) of the molecular complex is essential for its unidirectional rotation $[56,128]$. 
At equilibrium condensed phase, the rotation of motor has equal probabilities of forward and backward motions based on the principle of detailed balance. Therefore, unidirectional motion results only when the motor is at nonequilibrium state because of some chemical or electrochemical force; hence the motion of motor is a dissipative process taking place at nanoscale and affected by thermal fluctuations [128]. The cycle of the motor corresponds to the full revolution $\left(360^{\circ}\right)$ with $s=6$ substeps and to the hydrolysis of three ATP molecule [50,126-128]

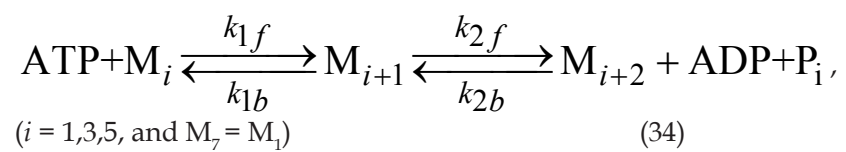

where $\mathrm{M}_{i}$ shows the six successive states of hydrolytic motor [50]. The chemical affinity generates fluctuating flows, which can be the rate of chemical reaction, or the velocity of a linear molecular motor, or the rotation rate of a rotary motor. According to the fluctuation theorem [50], the probability of backward substeps (s) is given by $P(-s)=P(s) \exp \left[-s A /\left(6 k_{B} T\right)\right]$ where the affinity $A$ is $[50,128]$

$$
A=-3 \Delta G^{o}+3 k_{B} T \ln \left(\frac{[\mathrm{ATP}]}{[\mathrm{ADP}]\left[\mathrm{P}_{\mathrm{i}}\right]}\right),
$$

with the standard free enthalpy of hydrolysis

$$
\Delta G^{o}=\Delta G_{\mathrm{ATP}}^{o}-\Delta G_{\mathrm{ATP}}^{o}-\Delta G_{\mathrm{P}_{\mathrm{i}}}^{o} \cong 50 \mathrm{pN} \mathrm{nm} \text { at } \mathrm{pH}
$$

$=7$ and $T=23^{\circ} \mathrm{C}[129]$. Equilibrium concentrations obey

$$
\left(\frac{[\mathrm{ATP}]}{[\mathrm{ADP}]\left[\mathrm{P}_{\mathrm{i}}\right]}\right)_{\mathrm{eq}}=\frac{k_{1 b} k_{2 b}}{k_{1 f} k_{1 f}}=e^{-\Delta G^{o} / k_{B} T}=4.8910^{-6} / \mathrm{M}
$$

Under physiological conditions, the concentrations are about [ATP] $\approx 10^{-3} \mathrm{M},\left([\mathrm{ATP}]_{\mathrm{eq}} \approx 4.8910^{-13}\right),[\mathrm{ADP}] \approx 10^{-4} \mathrm{M}$, and $\left[\mathrm{P}_{\mathrm{i}}\right] \approx 10^{-3} \mathrm{M}$, hence the motor runs in a highly nonlinear regime, that is far from equilibrium, with an affinity $A \geq 40 k_{B} T[50,128,129]$. In this regime, the fluctuation theorem shows that the backward steps are rare, and unidirectional motion can overwhelm erratic Brownian motion $[50,128]$. During the unidirectional motion the motor undergoes a cycle of intramolecular transformations, in which its three dimensional structure changes with time [50] leading to a temporal ordering as the feature of systems driven far from equilibrium. Some future applications of the fluctuation theorem to molecular machines may be single-molecule pulling experiments on RNA, DNA, proteins, and other polymers to determine their free-energy landscapes. The fluctuation theorem is satisfied along the thermodynamic branch (Figure1.) of near and far from equilibrium regions, and shows that the ratio of the probability of a forward rotation of the shaft to the probability of backward rotation determines the thermodynamic force, affinity, as the key information for the nonequilibrium thermodynamics of molecular motors [50,128].

\subsection{Application to molecular motor kinesin}

Kinesin is a large protein which can attach to a load on one end and has two heads on the other end, and performs an asymmetric hand on hand walk along a microtubule leading to effectively dragging the load against an external force $F$ and the viscous drag from the environment. Each step in this walk corresponds to a cycle. Therefore, kinesin converts chemical energy released by the hydrolysis of one ATP molecule into useful work. The amount of energy released by the hydrolysis of one ATP molecule is around $25 k_{B} T$, where $k_{B}=1.4$ $\times 10^{-23} \mathrm{~J} / \mathrm{K}$ is the Boltzmann constant and $T=300 \mathrm{~K}$ is the environment temperature [130]. At some point when the head of kinesin is free it has many more available states than when it is docked, and so it also has larger entropy. Therefore docking must be accompanied by an entropy decrease leading to a spatial order and heat loss $Q$ to the environment. The distance between the attachment sites on the microtubule is $\delta=8 \mathrm{~nm}$. Kinesin can exert a constant force, mostly independent of the opposing external force. Otherwise, the free head is caught up in the thermal Brownian motion generated by the environment: the free head can dock in the intended site, in which case the step is successfully completed, or else it may be dragged back to its initial position. The probability of a successful forward step over that of backward step is $[129,130]$

$$
\frac{P_{f}}{P_{r}}=\exp \left(\frac{\mathrm{d}}{2 k_{B} T}\left[F_{s t}-F\right]\right),
$$

where $F_{s t} \approx 7 \mathrm{pN}$ is the stalling force. Eq. (37) shows that the maximum work kinesin can do against the external force is $F_{s t} \delta \approx 13.3 k_{B} T$, which is close to half of the available energy of $25 k_{B} T$.

To obtain the free energy change associated to one step from observable data, the Crooks fluctuation theorem can be used. Assuming the macroscopic initial and final states 1 and 2 are the initial and final states, respectively in the kinesin cycle, and the external parameter $\lambda$ measures the progress of the molecule from one pair of docking sites to the next. A backward step implies as that the work ( $W$ $=F \delta$ ) is reversed. For the free energy $G$, the Clausius inequality implies $W \leq-\Delta G$ in any isothermal process. The system depends on some time dependent external parameter $\lambda$. Initially the system is at state 1 . If the $P_{f}$ is the probability that the system ends up in state 2 , giving out work $W$ and the $P_{r}$ is the probability that the system, now starting from state 2 , ends up in state 1 giving out work $-W$ when the evolution of $\lambda$ is reversed, the Crooks fluctuation theorem states that [131]

$$
\frac{P_{f}}{P_{r}}=\exp \left(\frac{-1}{k_{B} T}[\Delta G+W]\right) .
$$

The Crooks fluctuation theorem of Eq. (38) with the probability ratio given in Eq. (37) implies that

$\Delta G=\frac{-\mathrm{d}}{2}\left[F_{s t}+F\right]$,

where $\Delta G$ is the maximum work which may be extracted from the system at constant temperature. Ideally, all the energy available to the kinesin at the start of the cycle $\left(2 \delta F_{s t}=26.6 k_{B} T\right)$ is dissipated or goes to into the reversible work [130]. For isothermal docking we have $S_{\text {free }}-S_{\text {dock }}=(\delta / 2 T)[3 F s t-F]$, where $S_{\text {free }}$ and $S_{\text {dock }}$ are the entropies of the free head and docked states, respectively. Not all the available work is actually extracted: some of it is left to be dissipated as heat, for example by opposing the viscous drag or as excess kinetic energy to be absorbed by the docking site. Beside that, the cycle may fail, with the kinesin stepping backwards rather than forward. Therefore

the actual average work is $\langle W\rangle=F\langle\mathrm{~d}\rangle$, where $\langle\mathrm{d}\rangle$ is the average displacement given by [131]

$$
\langle\mathrm{d}\rangle=\mathrm{d} \tanh \left(\frac{\mathrm{d}}{4 k_{B} T}\left[F_{s t}-F\right]\right) .
$$

This analysis illustrates the estimation of the free energy change of 


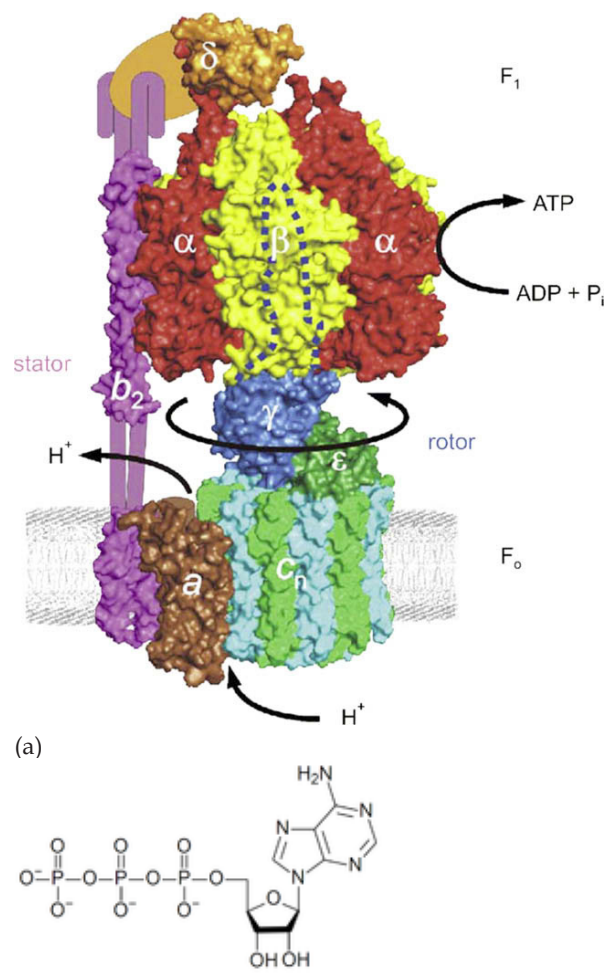

(b)

Figure 2. (a) ATPases are a class of enzymes that catalyze the synthesis and hydrolysis of adenosine triphosphate (ATP); (b) structural formula of ATP. The domain $\mathrm{F}_{\mathrm{O}}$ consists of one $\mathrm{a}$, two $\mathrm{b}$ and a ring of 9-15 subunits $\mathrm{c}$ depending on the species. The subunits $\mathrm{c}$ form a ring, connected to the domain $\mathrm{F}_{1}$ via the subunit $\varepsilon$ and then $\gamma$ and two subunits $b$ and $\delta$. Water-soluble $F_{1}$ domain has the subunits $\alpha_{3} \beta_{3} \gamma \delta \varepsilon$. Catalytic nucleotide-binding sites are formed by each of three subunits $\beta$ [125-127].

nonequilibrium dynamics of kinesin by using the Crooks fluctuation theorem.

\section{Bioenergetics}

Bioenergetics is concerned with the energy production, conservation, and conversion processes in living cells [41,51]. The clusters of orthologous gene database have identified 210 protein families involved in energy production and conversion; they show complex phylogenetic patterns that cause diverse strategies of energy conservation [132]. The respiration chain generates energy by the oxidation of reducing equivalents of nutrients (nicotinamide adenine nucleotides NADH and the flavin nucleotides FADH2), which is conserved as the ATP through the oxidative phosphorylation (OP) in the inner membrane of the mitochondria. Cytochrome $c$ oxidase, terminal enzyme of the chain reduces oxygen to water with four electrons from cytochrome $\mathrm{c}$ and four protons taken up from the matrix of mitochondria, and pumps protons from the matrix into the intermembrane space, causing an electrochemical proton gradient across the inner membrane, which is used by the $\mathrm{F}_{0} \mathrm{~F}_{1}$-ATPase to synthesize ATP. There is also another cycle (the $\mathrm{Q}$ cycle) around the cytochrome $b c 1$ complex, which causes substantial proton pumping. Synthesis of ATP is an endergonic reaction and hence, conserves the energy released during biological oxidation-reduction reactions. As Figure3a illustrates, respiration cycle drives the ATP production through the thermodynamically coupling mechanisms in the OP. On the other hand, photosynthesis, driven by light energy, also leads to production of ATP through electron transfer and photosynthetic phosphorylation. Hydrolysis of one mole of ATP is an exergonic reaction releasing $31 \mathrm{kJmol}$ at $\mathrm{pH}=7$. This energy drives various energy-dependent metabolic reactions and the transport of various ions such as $\mathrm{H}^{+}, \mathrm{K}^{+}$and $\mathrm{Na}^{+}$. Synthesizing of ATP is matched and synchro- nized to cellular ATP utilization:

$$
\mathrm{ADP}+\mathrm{P}_{\mathrm{i}}+n \mathrm{H}_{\text {in }}^{+}=\mathrm{ATP}+\mathrm{H}_{2} \mathrm{O}+n \mathrm{H}_{\text {out }}^{+} \text {, }
$$

where 'in' and 'out' denote two phases separated by a membrane, and $n$ is the ratio $\mathrm{H}^{+} / \mathrm{ATP}$, showing the level of transmembrane proton transport for each ATP to be synthesized by rotational catalysis in the $\mathrm{F}_{1}$ domain of mitochondrial $\mathrm{F}_{\mathrm{o}} \mathrm{F}_{1}$-ATPase [125-127] shown in Figure 2.

5.1. Linear nonequilibrium thermodynamics analysis of oxidative phosphorylation

In the last five decades, various new approaches in thermodynamics, non-equilibrium thermodynamics and network thermodynamics have been developed to understand and describe the bioenergetics $[21,31,41,51]$. These approaches are based on the irreversible character of flows and forces of an open system. Experimental investigations revealed that the linear nonequilibrium thermodynamics (LNET) formulations are capable of describing thermodynamically coupled flow and reaction processes of $\mathrm{OP}$, mitochondrial $\mathrm{H}^{+}$pumps, and $\left(\mathrm{Na}^{+}\right.$and $\left.\mathrm{K}^{+}\right)$-ATPase (see Figure $3 \mathrm{~b}$ ), because mainly due to enzymatic feedback $[12,31,32,44-48,125,126]$. Moreover, the LNET formulations do not require the detailed mechanism of the coupling. Kinetic descriptions and considerations, on the other hand, may lead to a loss of the generality characteristics of thermodynamic formulations, since the kinetics is based on specific models [44].

Stucki et al. [45] applied the LNET theory of linear energy converters using the following approximate representative linear phenomenological relations of the OP without a load $(J=0)$, and shown in Figure 3a:

$$
\begin{aligned}
& J_{o}=L_{o} X_{o}+L_{p o} X_{p}, \\
& J_{p}=L_{p o} X_{o}+L_{p} X_{p} .
\end{aligned}
$$

Here $J_{o}$ and $J_{p}$ are the oxygen flow rate and the ATP production rate, respectively, while $X_{o}$ and $X_{p}$ show the redox potential for oxidazible substances and the phosphate potential, respectively [31,41]. The independent flows and forces are identified by the entropy production

equation $\Phi=J_{o} X_{o}+J_{p} X_{p}>0$, so the cross coefficients, $L_{o p}$ and $L_{p 0^{\prime}}$ obey Onsager's reciprocal rules, which states that $L_{o p}=L_{p o}$. Stucki [133] and later Cairns et al. [134] experimentally proved the approximate linearity of reactions in OP within the range of phosphate potentials of practical interest $[31,46,133]$. The degree of coupling is defined by [135]

$$
q=\frac{L_{o p}}{\left(L_{p} L_{o}\right)^{1 / 2}} \quad 0<|q|<1,
$$

and indicates the extent of overall coupling of the ATP production driven by the respiration cycle in the inner membrane of mitochondria. By defining the phenomenological stoichiometry (the phenomenological stoichiometry $Z$ differs from the molecular stoichiometry in kinetics) by

$Z=\left(\frac{L_{o}}{L_{p}}\right)^{1 / 2}$

and by dividing Eq. (42) by Eq. (43), we obtain the flow ratio $j=J_{0} /$ $\left(J_{p} Z\right)$ in terms of the force ratio $x=\left(X_{o} Z / X_{p}\right)$ as follows

$$
j=\frac{x+q}{q z+1} .
$$


The efficiency of the linear energy conversion of OP may be defined as the ratio of output power to input power and in terms of the degree of coupling, and given by

$\mathrm{h}=-\frac{J_{p} X_{p}}{J_{o} X_{o}}=-\frac{x+q}{q+(1 / x)}$.

There are two stationary states called the static head (sh) in which the net rate of ATP vanishes and the level flow (lf) in which the phosphate potential vanishes. The value of efficiency reaches an optimum value between these two stationary states and is a function of the degree of coupling only

$\mathrm{h}_{o p t}=\frac{q^{2}}{\left(1+\sqrt{1-q^{2}}\right)^{2}}=\tan ^{2}(\mathrm{a} / 2)$

where $\mathrm{a}=\arcsin (q)$ [31,32,41,45,52,133-135]. The value of $x$ at $\eta_{\text {opt }}$ becomes [133]

$$
x_{\text {opt }}=-\frac{q}{1+\sqrt{1+q^{2}}} .
$$

The value of $q$ can be calculated from the measurements of oxygen flows at static head (sh) $\left(J_{o}\right)_{\text {sh }}$ and at uncoupled state (unc) $\left(J_{o}\right)_{\text {unc }}$ where the proton gradient vanishes and the respiration cycles is uncoupled from the OP [134]:

$$
q=\sqrt{1-\left(J_{o}\right)_{\mathrm{sh}} /\left(J_{o}\right)_{\mathrm{unc}}} .
$$

For example, the overall degree of thermodynamic coupling is higher for a rat liver mitochondrion $(0.955 \pm 0.021)$ than for brain $(0.937$ $\pm 0.026)$ or hearth $(0.917 \pm 0.037)$ [134].

Stucki [134 ] analyzed the required degrees of coupling of OP, when the ATP production is coupled to a load such as hydrolysis of the ATP (see Figure 3b), for the optimum production functions $f$ for the

ATP and output power given by $f=\tan ^{m}(\mathrm{a} / 2) \cos (\mathrm{a})$ [133] The optimum production functions and the associated constants are described in Table 3, while Figure 4 shows the effect of degree $q_{f}, q_{p}, q_{f}^{e c}$, and $q_{p}^{e c}[31,32,41]$.

of coupling on the optimum efficiency $[41,133,137,138]$. If the system has to maximize the ATP production at optimal efficiency then $q_{f}=0.786$. Instead, if the system has to maximize the power output at optimal efficiency, the output function $f=\left(J_{p} X_{p}\right)_{\text {opt }}$ occurs at $q_{p}=$ 0.91. If the additional constraint of efficient ATP synthesis (minimal energy cost) is imposed, then the economic ATP flow and economic

power output occur at $q_{f}^{e c}=0.953$ and $q_{p}^{e c}=0.972$, respectively $[31,133]$. The dynamic properties of OP based on the eigenvalue sensitivity analysis of a nonequilibrium thermodynamic model of $\mathrm{OP}$, for the parameters characteristic for liver of starved rats in vivo, indicates that the sensitivity of OP to a fluctuating ATP utilization is minimal at a degree of coupling $q=0.95$. This means that the phosphate potential is highly buffered with respect to fluctuating energy demands at the degree of coupling, which is very close to the val-

ue of $q_{f}^{e c}$, at which net ATP production of OP occurs at the minimal energy cost [52].

\subsection{Chemomechanical energy transduction}

A motor's n-dimensional state space having an energy $E$ may be described by a set of parameters $\left(a_{1}, a_{2},, a_{\mathrm{n}}\right)$ that represent variable structural elements, like the bond angles in a motor's carbon skeleton or amino acid chains $E\left(a_{1}, a_{2}, ., a_{\mathrm{n}}\right)$. These structures are dynamic; they fluctuate about an energy minimum upon motor biochemical transition, such as ATP binding and product(s) release. For example, ATP hydrolysis can occur through a spontaneous pathway in solution or through an enzyme-catalyzed pathway. Regardless of the pathways the free energy changes by $\Delta G_{\text {ATP. }}$. However, through an uncatalyzed pathway, $\Delta G_{\text {ATP }}$ is lost entirely as heat, where as through a motor catalyzed reaction pathway $\Delta G_{\text {ATP }}$ consists of heat $Q$ and external work $W_{\text {ext' }}$ while some other earlier models assume that $\Delta G_{\text {ATP }}$ is affected by internal work $W_{\text {int }}[24,31,117]$. A motor enzyme system is bathed within the ligand solution system where free ATP molecules are hydrolyzed and the forcing potential exists in the bulk ligand system not in the motor system. Thus, how a motor enzyme converts $\Delta G_{\text {ATP }}$ into mechanical work depends on how a motor enzyme interacts with the solution system. One simple approach used in solution thermodynamics may be to treat a biochemical transition as Markovian steps between discrete biochemical states, which are a collection of structural states within a given energy well and has a discrete energy level corresponding to the Boltzmann weighted average of the energies of all structural states. The chemical potential of

a biochemical state is $\mathrm{m}=\mathrm{m}^{\rho}+R T \ln \mathrm{P}_{M i}$, where $\mathrm{m}^{\rho}$ is the (a)

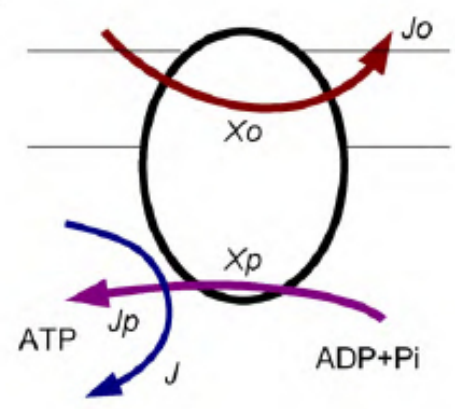

(b)

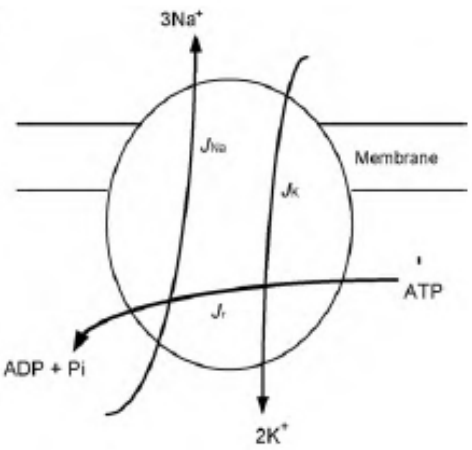

Fig. 3. (a) Energy coupling between respiration cycle and oxidative phosphorylation taking place in the innermembrane of mitochondria. Here $J_{e}$ and $J_{p}$ are the oxygen flow rate and the ATP production rate, respectively, while $X_{0}$ and $X_{p}$ show the redox potential for oxidazible substances and the phosphate potential, respectively. The flow $J$ represents a load coupled to ATP production, (b) Active transport of $\mathrm{Na}^{+}$and $\mathrm{K}^{+}$ions coupled to hydrolysis of ATP [31,44-46,51]. 
Table 3

Production functions with the consideration of conductance matching $[45,134,135]$

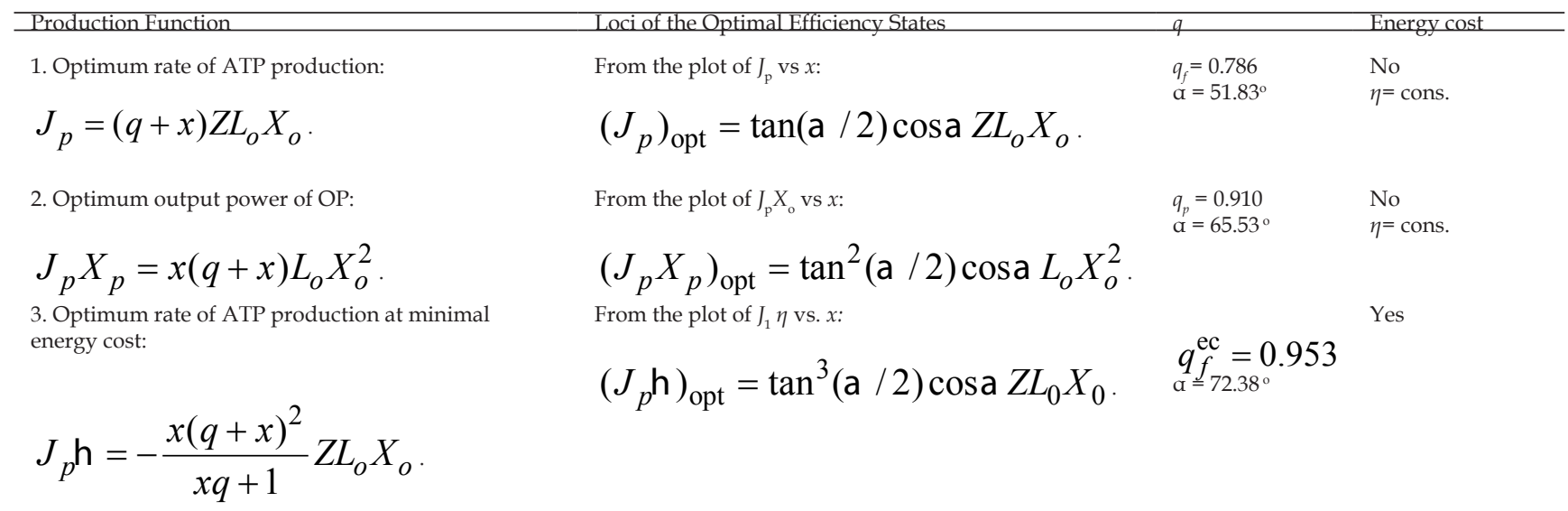

4. Optimum output power of OP at minimal energy cost:

$$
J_{p} X_{p} \mathrm{~h}=-\frac{x^{2}(q+x)^{2}}{x q+1} L_{o} X_{o}^{2}
$$

From the plot of $J_{1} X_{1} \eta$ vs $x$ :

$$
\left(J_{p} X_{p} \mathrm{~h}\right)_{\mathrm{opt}}=\tan ^{4}(\mathrm{a} / 2) \cos \mathrm{a} L_{o} X_{o}^{2} . \quad{\underset{\mathrm{a}}{\underline{p}_{76.34^{\circ}}}}_{\mathrm{ec}}=0.972^{\text {Yes }}
$$

standard chemical potential at standard temperature, pressure, ionic strength, $\mathrm{pH}$, etc., and $P_{M i}$ is the probability that motor occupies state $i$. The free energy of solution ligand system is

$$
\Delta G_{A T P}=(n \Delta \mathrm{m})=\mathrm{m}_{A D P}+\mathrm{m}_{P_{i}}-\mathrm{m}_{A T P}=\Delta G_{A T P}^{o}+R T \ln \left(\frac{[\mathrm{ADP}]\left[\mathrm{P}_{\mathrm{i}}\right]}{[\mathrm{ATP}]}\right)
$$

$$
n \Delta \mathrm{m}=n W_{\mathrm{int}}+W_{\text {ext }}+Q \text { or } n\left(\Delta \mathrm{m}-W_{\mathrm{int}}\right)=W_{\text {ext }}+Q
$$

Earlier motor models suggested that a motor's work producing mechanisms are formally separated from its enzymatic mechanisms, while new models suggest that a motor's working step can consist of compliant regions within the motor and track $\left(W_{\text {int }}\right)$, performed by that step in stretching motor and track elastic elements, and the

$\Delta \mathrm{m}$ is partitioned between the $W_{\text {int }}$ and $W_{\text {ext }}[117]$.

\section{Reaction-diffusion systems}

Thermodynamically coupled transport and rate processes are very common in biochemical cycles, and require a thorough analysis by accounting the induced flows by cross effects [139-144], such as the hydrolysis of ATP coupled to the processes transporting substrates and maintaining the essential thermodynamic forces in active transport illustrated in Figure 3b. For example, $\mathrm{Ca}^{+2}$-ATPase in the plasma membranes of most cells pumsp $\mathrm{Ca}^{+2}$ against a steep concentration gradient out of cytosol, while simultaneously counterports $\mathrm{H}^{+}$ ions [44].

In 1952, Turing proposed a hypothetical sequential route for cell differentiation, based on a reaction diffusion process [140], which was verified later [141]. Starting from a spatially uniform state Turing proved that stable inhomogeneities in "morphogen" concentration could spontaneously emerge through a diffusion-driven symmetry breaking instability $[48,78,142,143]$. The general concept behind Turing patterns involves a combination of short-range activation and large-range inhibition. Stirrer and Dawson [142] showed the existence of cell-sized Turing patterns in glycolysis, using real- istic parameter values and equal diffusion coefficients of ATP and ADP. They showed that a model of the enzymatic step catalyzed by phosphofructokinase (PFK), a step which is responsible for the appearance of homogeneous oscillations in the glycolytic pathway, displays Turing patterns with an intrinsic length-scale $(10 \mu \mathrm{m})$ that is smaller than a typical cell size. All the parameter values are fully consistent with classic experiments on glycolytic oscillations with equal diffusion coefficients for ATP and ADP. They identified the enzyme concentration and the glycolytic flux as the possible regulators of the pattern. The emergence of these patterns can be traced back to the differential interactions of ATP and ADP with PFK and its complexes. The key enzymatic step responsible for glycolytic oscillations may also provide a robust mechanism for the formation of steady state inhomogeneities in the concentration of ATP and ADP at the cellular and supracellular level [142]. The patterns can fit inside a tvpical cell and that the time it takes for the patterns to form

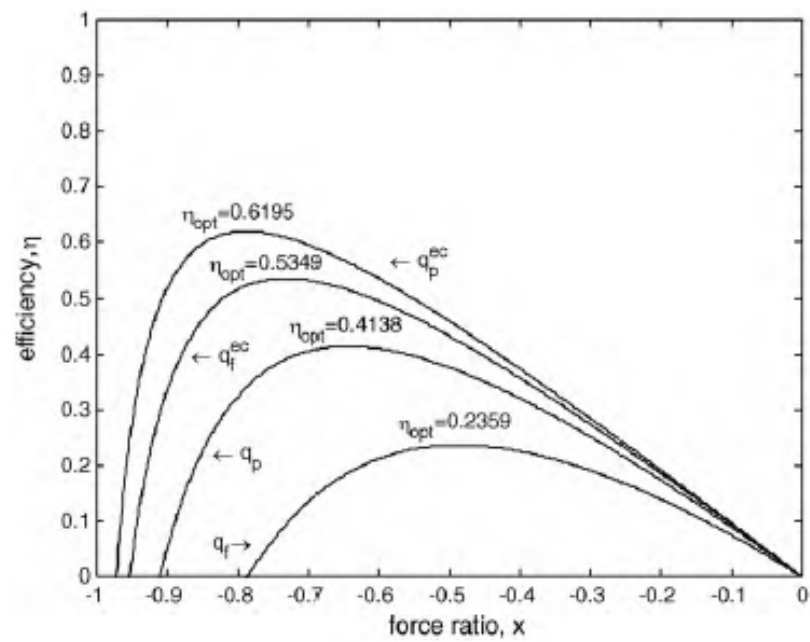

Fig. 4. Efficiency vs, force ratio at various levels of thermodynamic couplings. The change of efficiencies $\eta$. given in Eq. (47), in terms of flow ratio $x$ and for the degrees of coupling $q_{f}, q_{p}, q_{f}^{* c}$, and $q_{p}^{* c}[31,32,41]$. 
reaction between a substrate (S) and a product $(\mathrm{P}) \mathrm{S} \underset{k_{b}}{\stackrel{k_{f}}{\rightleftarrows}} \mathrm{P}$.

This type of reaction system is common in chemical and biological systems, such as unimolecular isomerization [48], enzyme kinetics [31], and racemization of molecules with mirror-image structures [48]. The well known balance equations are

$$
\frac{\partial C_{S}}{\partial t}=\nabla \cdot \mathbf{J}_{S}+v_{S} J_{r}
$$

$\frac{\partial C_{P}}{\partial t}=\nabla \cdot \mathbf{J}_{P}+v_{P} J_{r}$

$$
\rho c_{p} \frac{\partial T}{\partial t}=\nabla \cdot \mathbf{J}_{q}+\left(-\Delta H_{r}\right) J_{r}
$$

where $\mathbf{J}_{\mathbf{i}}$ is the vector of mass flows for species $i, \mathbf{J}_{\mathbf{q}}$ the vector of re-

duced heat flow $\mathbf{J}_{q}=\mathbf{q}-\sum_{i=1}^{n} \mathbf{J}_{i} H_{i}, \mathbf{q}$ is the total heat flow, $H_{i}$ the partial molar enthalpy of species $i$, and $\Delta H_{r}$ the heat of reaction, $C_{i}$ the concentration of species $i, c_{p}$ the heat capacity, and $\rho$ is the den-

sity. The reaction velocity is $\frac{C_{S}}{v_{S} d}=\frac{\mathbb{C}_{P}}{v_{P} d}=J_{r}$, and the parameters $v_{S}$ and $v_{p}$ are the stoichiometric coefficients, which are negative for reactants $\left(v_{S}=-1\right)$.

Fick's law is incomplete when used to describe the flows of charged particles. The migrational flows of ions are driven by the electric field which is generated internally by the flows of ions of unequal mobilities [144,145]. The electric field slows down the more mobile ions and speeds up the less mobile ions in order to maintain electroneutrality along the diffusion path. Acidic solutions containing ions with widely different mobilities generate rather large electric fields; for example, the mobility of the $\mathrm{H}^{+}$ions is typically five to ten times larger than the mobilities of the other ions [144]. Therefore, reactions consuming or producing $\mathrm{H}^{+}$ions produce sharp gradients in concentrations of $\mathrm{H}^{+}$ions and hence substantial electric fields. This would provide driving forces for the coupled diffusion of other ions in the solution. The Nernst-Planck equation include the coupled induced flows of the ionic species $i$ beside the pure-diffusion flows

$$
\mathbf{J}_{i}=-D_{i e} \nabla C_{i}+(F / R T) z_{i} D_{i e} C_{i} \mathbf{E} .
$$

where $E$ is the electric field, $F$ the Faraday constant, $R$ the gas constant, $T$ the temperature, $z_{S}$ the charge number of species $S$, and $D_{i e}$ is the effective diffusivity for component $i$. The Nernst-Planck equation does not include changes in the ionic diffusion coefficients caused by electrophoresis and changes in the driving forces caused by the variation in the ionic activity coefficients in the solution. However, the correction due to Eq. (56) is mostly satisfactory, especially in dilute solutions of univalent electrolytes, and when the diffusion coefficients $D_{S e}$ of solution species are estimated from ionic conductivities or deduced from diffusion measurements on binary solutions of single electrolytes [144].

Chemical reaction-diffusion systems with heat effects represent open and nonequilibrium systems with thermodynamic forces of temperature gradient, concentration gradient, and affinity. By using the Gibbs-Duhem equation at constant temperature and pres- sure $\left(C_{S} \nabla \mu_{S}+C_{P} \nabla \mu_{P}=0\right)$, and no volume flow condition $\left(\mathbf{J}_{S} V_{S}+\mathbf{J}_{P} V_{P}=0\right.$ ), where $V_{i}$ is the partial molar volume of species $i$, the local rate of entropy production of such a system is $[21,59,146,147]$ :

$\Phi=-\mathbf{J}_{q}\left(\frac{1}{T^{2}}\right) \nabla T-\mathbf{J}_{S} \frac{1}{T} \lambda_{S} \nabla C_{S}+J_{\underset{(57)}{ }} \frac{A}{T} \geq 0 ，$

where

$\lambda_{S}=\left(1+\frac{C_{S}}{C_{P}}\right)\left(\frac{\partial \mu_{S}}{\partial C_{S}}\right)_{T, P}$ for $\left(V_{S} \approx V_{P}\right)$

Equation (57) identifies the independent conjugate flows $J_{i}$ and forces $X_{k}$ to be used in the linear phenomenological equations when the system is in the vicinity of global equilibrium. Linear flow-force relations are valid when the Gibbs free energy ranges less than 1.5 $\mathrm{kJ} / \mathrm{mol}$ for chemical reactions $[31,32,48,49]$. The formalism of LNET can be used in wider ranges (over a $7 \mathrm{~kJ} \mathrm{~mol}^{-1}$ ) than usually expected with an error in the reaction velocity less than $15 \%$ [28,31,45-49] for some reactions and for selected biological pathways. The linear phenomenological equations become $[21,41,45,82]$

$$
\begin{aligned}
& \mathbf{J}_{S}=-D_{S, e} \nabla C_{S}-D_{T, e} \nabla T+\mathbf{L}_{S r} \frac{A}{T} \\
& \mathbf{J}_{q}=-D_{D, e} \nabla C_{S}-k_{e} \nabla T+\mathbf{L}_{q r} \frac{A}{T} \\
& J_{r}=-\left.\mathbf{L}_{r S} \frac{1}{T}\right|_{S} \nabla C_{S}-\mathbf{L}_{r q} \frac{1}{T^{2}} \nabla T+\frac{k_{f} C_{S, e q}}{R} \frac{A}{T},
\end{aligned}
$$

where $D_{T, e}$ and $D_{D, e}$ are related to the thermal diffusion and the Dufour effect, respectively and represent cross effects due to thermodynamic coupling, and $k_{e}$ is the effective thermal conductivity $[146,147]$. Onsager's reciprocal relations states that $L_{i k}=L_{k j}(i \neq k)$ if $J_{i}$ and $J_{k}$ have the same parity under time reversal, and $L_{i k}=-L_{k i}$ if $J_{i}$ and $J_{k}$ have the opposite parity. For the nonvanishing cross coefficients $L_{i k}$, all the forces contribute for each flow. In the absence of pertinent symmetries or invariances, all types of cross-couplings are possible. If the structure of the system is invariant with respect to some or all of the orthogonal transformations, then the invariance will eliminate certain cross-couplings and their cross-coefficients will vanish. Here, Eqs. (58) to (60) take into account the thermodynamic couplings between vectorial processes and between vectorial and scalar processes, which is possible in an anisotropic medium according to the Curie-Prigogine principle [48]. For example, in active transport in biological cells, the hydrolysis of ATP is coupled with the flow of sodium ions outside of the cell (Figure $3 \mathrm{~b}$ ) and the flow direction is controlled by the structure of the membrane and coupling mechanisms in mitochondria. The medium may be locally isotropic, although it is not spatially homogenous. In this case, the coupling coefficients are associated with the whole system $[31,48]$.

If we consider the change of affinity with time at constant temperature and pressure, we have

$\frac{d}{d}=\left(\frac{\partial A}{\partial C_{S}}\right)_{T, P} \frac{\mathbb{C}_{S}}{\boldsymbol{d}}$ 
Itis possibleto split the $d C_{s}$ intotwo parts: $\mathbb{C}_{S}=d_{e} C_{S}+d_{i} C_{S}$ where $d_{e} C_{S}$ results from the exchange with the surrounding, while $d_{i} C_{S}$ due to a chemical reaction. The reaction velocity is

$d_{i} C_{S} / \boldsymbol{d}=v_{S} J_{r}$. With these relations Eq. (61) yields

$$
\frac{d}{d}=\left(\frac{\partial A}{\partial C_{S}}\right)_{T, P} \frac{d_{e} C_{S}}{d}-J_{\delta}\left(\frac{\partial A}{\partial C_{S}}\right)_{T, P} .
$$

Eq. (62) shows that affinity changes by rate of matter exchanged with the surrounding and chemical reaction velocity; therefore, the terms on the right of Eq. (62) may counter balance each other so the affinity reaches a stationary value, which may lead to a specific behavior in the evolution of the whole system $[46,48]$.

By inserting Eqs. (58) to (60) into Eqs. (53) to (55), we may describe the thermodynamically and mathematically coupled system of chemical reaction and heat and mass flows in one-dimensional form $[21,31,32,146]$ by:

$$
\frac{\partial \mathbf{q}_{S}}{\partial \mathrm{t}}=\frac{\partial^{2} \mathbf{q}_{S}}{\partial z^{2}}+\mathrm{e} \frac{\partial^{2} \mathbf{j}}{\partial z^{2}}+\frac{\mathrm{s}}{\mathbf{j}^{2}} \frac{\partial \mathbf{j}}{\partial z}-A^{*} \operatorname{Da}_{S} \mathbf{q}_{S, e q} \exp \left[\mathbf{g}_{f}\left(1-\frac{1}{\mathbf{j}}\right)\right]
$$

$\frac{1}{, L e} \frac{\partial \mathbf{j}}{\partial \mathrm{t}}=\frac{\partial^{2} \mathbf{j}}{\partial z^{2}}+\mathrm{w} \frac{\partial^{2} \grave{e}_{S}}{\partial z^{2}}-\frac{\mathrm{k}}{\mathrm{j}} \frac{\partial \mathrm{q}_{S}}{\partial z}+\ddot{\mathbf{e}}^{*} \mathrm{Da}_{S} \mathrm{~b}_{S, e q} \exp \left[\mathrm{g}_{f}\left(1-\frac{1}{\mathrm{j}}\right)\right]$

where

$$
\begin{aligned}
& \mathrm{e}=\frac{D_{T, e} T_{S}}{D_{S, e} C_{S s}}, \quad \mathrm{w}=\frac{D_{D, e} c_{s}}{k_{e} T_{s}}, \quad \mathrm{~s}=\frac{\left[L_{r q}+L_{S r}\left(-\Delta H_{r}\right)\right] L}{T_{s} D_{S, e} C_{S s}}, \quad \mathrm{k}=\frac{\left[L_{r q}+L_{S r}\left(-\Delta H_{r}\right)\right] C_{S s} L l_{S}}{k_{e} T_{s}^{2}} \\
& \text { and } \\
& A^{*}=\left(\frac{\mathrm{g}_{b}-\mathrm{g}_{f}}{\mathrm{j}}\right)+\ln \left(\frac{\mathrm{q}_{S}}{K\left[a_{1}+a_{2}\left(1-\mathrm{q}_{S}\right)\right]} ;\right. \\
& z=\frac{y}{L}, \mathrm{t}=\frac{D_{S, e} t}{L^{2}} \\
& \mathrm{Da}_{S}=\frac{L^{2} k_{o} \exp \left(E_{f} / R T_{s}\right)}{D_{S, e}}, \mathrm{~g}_{f}=\frac{E_{f}}{R T_{S}}, \mathrm{~g}_{b}=\frac{E_{b}}{R T_{S}}, \mathrm{Le}=\frac{k_{e} / \mathrm{r} C_{p}}{D_{S, e}} \\
& \mathrm{q}_{S}=\frac{C_{S}}{C_{S S}}, \mathrm{q} P=\frac{C_{P}}{K C_{S S}}, a_{1}=\frac{C_{P S}}{K C_{S S}}, a_{2}=\frac{D_{S, e}}{K D_{P, e}}, \\
& \quad \mathrm{j}=\frac{T}{T_{S}}, \mathrm{~b}=\frac{\left(-\Delta H_{r}\right) D_{S, e} C_{S s}}{\left(-\mathrm{n}_{S}\right) k_{e} T_{S}} .
\end{aligned}
$$

The parameters $\varepsilon, \sigma, \omega$, and $\mathrm{k}$ are associated with the cross coefficients and hence control the induced flows because of the coupled reaction-transport system. Specifically, the $\varepsilon$ and $\omega$ control the coupling between mass and heat flows, while the $\sigma$ and $\mathrm{\kappa}$ control the coupling between the chemical reaction and mass flow, and chemical reaction and heat flow, respectively. Therefore, induced effects due to various coupling phenomena can increase the possibility that the system may evolve to multiple states and diversify its behavior
[6]. Accuracy of the solutions obtained from Eqs. (63) and (64) depends on the reliable data, such as the effective transport coefficients and cross coefficients. Some degrees of imperfections due to parallel pathways of reaction or intrinsic uncoupling within the pathway itself may lead to leaks and slips in mitochondria $[45,48]$. Some special cases of coupled phenomena may be: (1) Coupled phenomena at stationary state, (2) No coupling between the heat flow and chemical reaction: $L_{r q}=L_{q r}=0$, and (3) No coupling between the mass flow and chemical reaction: $L_{r S}=L_{S r}=0[82,146]$.

During a diffusion-controlled reaction, matter may be transported through an interface, which separates the reactants and the product. The progress of the reaction may be affected by the morphology of the interface with complicated structure, which controls the boundary conditions for the transport problem. Morphological stability of interfaces in nonequilibrium systems may lead to self-organization and/or pattern-formation in biological, physical, and chemical systems [48].

Since the dynamic behavior of a reaction-transport system may be more apparent with the state-space diagrams, the temperature and concentration profiles may be replaced with the spatial

integral averages obtained from $\mathrm{q}^{\prime}(\mathrm{t})=\int_{0}^{1} \mathrm{q}(z, \mathrm{t}) d z$ and

$\mathbf{j}{ }^{\prime}(\mathrm{t})=\int_{0}^{1} \mathbf{j}(z, \mathrm{t}) d z$. Some results of approximate solutions

to mathematically and thermodynamically coupled equations of (63) and Eq. (64) and the state-space diagrams are given elsewhere $[82,146]$.

\section{Conclusions}

Component biology of a single macromolecule and systems biology of biochemical cycles represent nonequilibrium, stochastic, coupled, open, and dissipative structures with diverse of spatial and time scales. The data collected from experiments on genomics and metabolomics help identifying biochemical reaction mechanisms, the functions of individual molecules, and their interactions in regulatory biochemical cycles and networks. The self-organizing processes and certain functions are linked in any scale in biology; they are dissipative and operate under various constraints, hence they are maintained by continuous supply of outside and inside material, energy, and information. Any modeling approach should take into account the stochastic, self organizing, and dissipative nature of biochemical cycles by focusing on their collective behaviors and principles. The fluctuation theorems describe how irreversible macroscopic behavior evolves from time-reversible microscopic dynamics as either the observation time or the system size increases, and entropy production can be related to the dynamic randomness of the stochastic systems. Modeling approaches for reaction-diffusion systems with heat effects can also describe some of the thermodynamically coupled transport and rate processes of biochemical cycles. On the other hand, the distance from global equilibrium is another important controlling parameter on the behavior and evolution of biological systems and should be considered in the modeling. However, many of these approaches are in their early stages of their development and no single computational or experimental technique is able to span all the relevant and necessary spatial and temporal scales. Wide range of experimental and novel computational techniques with high accuracy, precision, coverage, and efficiency are necessary for understanding biochemical cycles and networks, enzyme kinetics, and molecular motors.

References

[1] L. Chistoserdova, S.W. Chen, A. Lapdius, M.E. Lidstrom, Methylotrophy in Methylobacterium extorquens AM1 from genomic point of view, J. Bacteriol. 185 (2003) 2980

[2] F.S. Collins, E.D., Green, A.E., Guttmacher, M.S. Guyer, A vision for the future of genomics research, Nature 422 (2003) 835

[3] B.N. Kholodenko, H.V. Westerhoff, (Eds.), Metabolic Engineering in the Post Genomic Era, Norfolk, Horizon Bioscience, 2004. 
[4] J.L. Reed, T.D. Vo, C.H. Schilling, B.O. Palsson, An expanded genome scale model of Escherichia coli K-12, Genome Biol. 4 (2003) R54.1

[5] H. Kitano, Systems biology: A brief overview, Science 295 (2002) 1662

[6] G. Nicolis, I. Prigogine, Self-Organization in Non-Equilibrium Systems: From Dissipative Structure to Order Through Fluctuations, Wiley, New York, 1977.

[7] F. Backouche, L. Haviv, D. Groswasser, A. Bernheim-Groswasser, A. Active gels: dynamics of patterning and self-organization, Phys. Biol. 3 (2006) 264

8. B.S. Glick, Let there be order, Nature Cell Biol. 9 (2007) 130.

9] S. Camazine (Ed.), J-L. Deneubourg (Ed.), N.R. Franks (Ed.), J. Sneyd (Ed.), G. Theraulaz (Ed.), E. Bonabeau (Ed.) Self-Organization in Biological Systems, Princeton Univ. Press, 2001.

[10] L. Hood, Systems biology: integrating technology, biology, and computation, Mechanism Ageing Develop. 124 (2003) 9.

[11] P. Ao, Metabolic network modeling: Including stochastic effects, Comp. Chem. Eng. 29 (2005) 2297.

[12] N. Dan, Understanding dynamic disorder fluctuations in single-molecule enzymatic reactions, Curr. Opin. Colloidal Interface Sci. 12 (2007) 314.

[13] J. Elf, M. Ehrenberg, Spontaneous separation of bistable biochemical systems into spatial domains of opposite phases, Syst. Biol. 1 (2004) 230.

[14] B. Han, J. Wang, Quantifying robustness and dissipation cost of yeast cell cycle network: the funneled energy landscape perspectives, Biophys. J. 92 (2007) 3755 [15] P. Ao, Potential in stochastic differential equations: Novel construction, J. Phys. A37 (2004) L25.

[16] A. Arkin, J. Ross, H.H. McAdams, Stochastic kinetic analysis of developmental pathway bifurcation in phage lambda-infected Escherichia coli cells, Genetics 149 (1998) 1633.

[17] G.S. Ayton, G.A. Voth, Multiscale simulation of transmembrane proteins, J. Struct. Biol. 157 (2007) 570.

[18] K. Kruse, F. Jülicher, Oscillations in cell biology, Curr. Opin. Cell Biol. 17 $2005)$

[19] E.J. Crampin, S.Schnell, P.E. McSharry, Mathematical and computational techniques to deduce complex biochemical reaction mechanisms, Prog. Biophys. Mol. Biol. 86 (2004) 77.

[20] Y. Demirel, S.I. Sandler, Nonequilibrium thermodynamics in engineering and science, J. Phys. Chem. B 108 (2004) 31.

[21] Y. Demirel, Nonequilibrium Thermodynamics: Transport and Rate Processes in Physical, Chemical and Biological Systems, second ed., Elsevier, Amsterdam, 2007.

[22] T. Surrey, F. Nédélec, S. Leibler, E. Karsenti, Physical properties determining self-organization of motors and microtubules, Science 292 (2001) 1167.

[23] E. Karsenti, Self-organization in cell biology: a brief history, Nature Reviews 9 (2008) 255.

[24] T.L. Hill, Free Energy Transduction and Biochemical Cycle Kinetics, Springer, New York, 1989.

[25] D.A. Beard, H. Qian, S.D. Liang, Energy balance for analysis of complex metabolic networks, Biophys. J. 83 (2002) 79.

[26] D. J. Evans, D. J. Searles, The fluctuation theorem, Adv. Phys. 51 (2002) 1529.

[27] D.M. Carberry J.C. Reid, G.M.Wang, E.M. Sevick, D.J. Searles, D.J. Evans,

Fluctuations and irreversibility: An experimental demonstration of a second-law-

like theorem using a colloidal particle held in an optical trap, Phys. Rev. Let. 92 (2004) 140601-1.

[28] P. Gaspard, Hamiltonian dynamics, nanosystems, and nonequilibrium statistical mechanics, Physica A 369 (2006) 201

[29] C. Bustamante, J. Liphardt, F. Ritort, The nonequilibrium thermodynamics of small systems, Phys. Today, July (2005) 43.

[30] H. Qian, D.A. Beard, Thermodynamics of stoichiometric biochemical networks far from equilibrium, Biophys. Chem. 114 (2005) 213.

[31] R.S. Caplan, A. Essig, Bioenergetics and Linear Nonequilibrium Thermodynamics. The Steady State, (Reprint), Harvard University Press, New York, 1999.

[32]J.W.Stucki, Thethermodynamic-bufferenzymes, Eur.J. Biochem.109(1980) 257

[33] R. Moreno-Bote, J. Rinzel, N. Rubin, Noise-induced alternations in an attractor network model of perceptual bistability, J. Neurophys. 98 (2007) 1125.

[34] J.D. Murray, Mathematical Biology II: Spatial Models and Biomedical Applications, third ed., Springer, New York, 2003.

[35] T. Misteli, The concept of self-organization in cellular architecture, J. Cell Biol.

[36] S. Kjelstrup, D. Bedeaux, Non-equilibrium Thermodynamics of Heterogeneous Systems Series on Advances in Statistical Mechanics, World Scientific, New York, 2008.

[37] J. Tabony, Historical and conceptual background of self-organization by reac-

[38] M. Vellela, H. Qian, Stochastic dynamics and non-equilibrium thermodynamics of a bistable chemical system: the Schlögl model revisited, J. R. Soc. Interface 1 (2009) 16.

[39] D. Reguera, J.M. Rubi, J.M.G. Vilar, The mesoscopic dynamics of thermodynamic systems, J. Phys. Chem. B 109 (2005) 21502.

[40] H. Qian, Open-system nonequilibrium steady-state: statistical thermodynamics, fluctuations and chemical oscillations, J. Phys. Chem. B 110 (2006) 15063.

[41] Y. Demirel, S.I. Sandler, Thermodynamics of bioenergetics, Biophys. Chem. 97 (2002) 87.

[42] H. Qian, Entropy production and excess entropy in nonequilibrium steadystate of single macromolecules, Phys. Rev. E 65 (2002) 021111.

[43] J.-L. Luo, N. Zhao, B. Hu, Effect of critical fluctuations to stochastic thermodynamic behavior of chemical reaction systems at steady state far from equilibrium, Phys. Chem. Chem. Phys. 4 (2002) 4149.
[44] A.R. Waldeck, K. van Dam, J. Berden, P.W. Kuchel, A nonequilibrium thermodynamics model of reconstituted Ca ${ }^{2+}$-ATPase, Eur. Biophy. J. 27 (1998) 255-262. [45] J.W. Stucki, M. Compiani, S.R. Caplan, Efficiency of energy conversion in model biological pumps optimization by linear nonequilibrium thermodynamic relations, Biophys. Chem. 18 (1983) 101.

[46] K.J.Rothschild, S.A. Ellias, AQ. Essig, H.E. Stanley, Nonequilibrium linear behavior of biological systems. Existence of enzyme-mediated multidimensional inflection points, Biophys. J. 30 (1980) 209.

[47] A.S. Cukrowski, A. Kolbus, On validity of linear phenomenological nonequilibrium thermodynamics equations in chemical kinetics, Acta Physica Polonica B 36 (2005) 1485

[48] D. Kondepudi, I. Prigogine, Modern Thermodynamics; From Heat Engines to Dissipative Structures, Wiley, New York, 1999.

[49] I. Prigogine, Introduction to Thermodynamics of Irreversible Processes, third ed. Wiley, New York, 1967.

[50] D. Andrieux, P. Gaspard, Fluctuation theorems and the nonequilibrium thermodynamics of molecular motors, Phys. Rev. E 74 (2006) 011906-1.

[51] R.J.P. Williams, Molecular and thermodynamic bioenergetics, Proceedings of the first joint German/British Bioenergetics Meeting, Wiesbaden, Germany, 2024 March 2005.

[52] J.W. Stucki, Non-equilibrium thermodynamic sensitivity of oxidative phosphorylation, Proc. Biol. Sci. 244 (1991) 197.

[53] E.H. Lemus, Non-equilibrium thermodynamics of gene expression and transcriptional regulation, J.Non-Equilib. Thermodyn. 34 (2009) 371.

[54] D. Russel, K. Lasker, J. Phillips, D. Schneidman-Duhovny, J.A. VelazquezMiriel, A. Sali, The structural dynamics of macromolecular processes, Curr. Opin. Cell Biol. 21 (2009) 97.

[55] J. Paulsson, Models of stochastic gene expression, Phys. Life Rev. 2 (2005)

[56] A. Goldbeter, Oscillations and waves of cyclic AMP in Dictyostelium: a prototype for spatio-temporal organization and pulsatile intercellular communication, Bull. Math. Biol. 68 (2006) 1095

[57] A.M. West, R. Elber, D. Shalloway, Extending molecular dynamics time scales with milestoning: example of complex kinetics in a solvated peptide, J. Chem. Phys. 126 (2007) 145104

[58] DOE/SC-0121 New Frontiers in Characterizing Biological Systems: Report May 2009 Workshop, U.S. Department of Energy Office of Science (genomicscience.energy.gov/characterization/)

[59] M.L. Martins , S.C Ferreira, M.J. Vilela, Multiscale models for biological systems, Curr. Opin. Colloid Interface Sci. (2009), doi:10.1016/j.cocis.2009.04.004.

[60] I.M. Cristea, R. Williams, B.T. Chait, M.P. Rout, Fluorescent proteins as proteomic probes, Mol. Cell Proteomics 4 (2005)1933.

[61] C. Joo, H. Balci, Y. Ishitsuka, C. Buranachai, T. Ha, Advances in single-molecule fluorescence methods for molecular biology, Annu. Rev. Biochem. 77 (2008) 51. [62] J.R. Moffitt, Y.R. Chemla, D. Izhaky, C. Bustamante, Differential detection of dual traps improves the spatial resolution of optical tweezers, PNAS 103 (2006) 9006.

[63] S.R. McGuffee, A.H. Elcock, Atomically detailed simulations of concentrated protein solutions: the effects of salt, $\mathrm{pH}$, point mutations, and protein concentration in simulations of 1000- molecule systems, J. Am. Chem. Soc. 128 (2006) 12098

[64] A.W. Van Wynsberghe, Q. Cui, Interpreting correlated motions using normal mode analysis, Structure 14 (2006)1647.

[65] N. A. Sinitsyn, N. Hengartnerb, I. Nemenmana, Adiabatic coarse-graining and simulations of stochastic biochemical networks, PNAS 106 (2009) 10546.

[66] E.O. Voit, Computational Analysis of Biochemical Systems. A Practical Guide for Biochemists and Molecular Biologists, Cambridge University Press, Cambridge, 2000.

[67] H. Qian, D.A. Beard, S.D. Liang, Stoichiometric net work theory for nonequilibrium biochemical systems, Eur. J. Biochem. 270 (2003) 415.

[68] B.O. Palsson, N.D. Price, J.A. Papin, Development of network-based path way definitions: The need to analyze real metabolic networks, Trends Biotechnol. 21 (2003) 195

[69] W. Min, L. Jiang, J. Yu, S.C. Kou, H. Qian, X.S. Xie, Nonequilibrium Steady State of a Nanometric Biochemical System: Determining the Thermodynamic Driving Force from Single Enzyme Turnover Time Traces, Nano Let. 5 (2005) 2375 [70] M.A. Lomholt, P.L. Hansen, L. Miao, A general theory of nonequilibrium dynamics of lipid-protein fluid membranes, Eur. Phys. J. E 16 (2005) 439.

[71] D.A. Beard, E. Babson, E. Curtis, H. Qian, Thermodynamic constraints for biochemical networks, J. Theor. Biol. 228 (2004) 327.

[72] R. Nigam, S. Liang, Algorithm for perturbing thermodynamically infeasible metabolic networks, Comp. Biol. Med. 37 (2007) 126.

[73] R. Nigam, S. Liang, A Pivoting Algorithm for Metabolic Networks in the Presence of Thermodynamic Constraints, Computational Systems Bioinformatics, Stanford, 2005.

[74] W. Liebermeister, E. Klipp, Bringing metabolic networks to life: convenience rate law and thermodynamic constraints, Theor. Biol. Med. Model. 3 (2006) 41.

[75] N.D. Price, I. Famili, D.A. Beard, B.O. Palsson, Extreme pathways and Kirchhoff's second law, Biophys. J. 83 (2002) 2879.

[76] D.A. Beard, H. Qian, Relationship between thermodynamic driving force and one-way fluxes in reversible processes, PLoS One 2 (2007) e144.

[77] R.E. Carazo-Sala, P. Nurse, Self-organization of interphase microtubule arrays in fission yeast, Nature Cell Biol. 8 (2006) 1102. 
[78] J.M Chung, E.Peacock-Lopez, Bifurcation diagrams and Turing patterns in a chemical self-replicating reaction-diffusion system with cross diffusion, J. Chem. Phys. 127 (2007) 174903-1.

[79] H. Qian, Phosphorylation energy hypothesis: open chemical systems and their biological functions, Annu. Rev. Phys. Chem. 58 (2007) 113

[80] Q. Jin, C.M. Bethke, Kinetics of electron transfer through the respiratory chain, Biophys. J. 83 (2002) 1797.

[81] F. Schlögl, Chemical reaction models for nonequilibrium phase transition, $\mathrm{Z}$. Physik. 253 (1972) 147.

[82] Y. Demirel, Non-isothermal reaction diffusion system with thermodynamically coupled heat and mass transfer, Chem. Eng. Sci. 61 (2006) 3379.

[83] H. Qian, Cycle kinetics, steady state thermodynamics and motor-a paradigm for living matter physics, J. Phys. Condens. Matter 17 (2005) S3783.

[84] B. Pfeuty, K. Kaneko Minimal requirements for robust cell size control in eukaryotic cells, Phys. Biol. 4 (2007) 194.

[85] N. Barkai, S. Leibler, Robustness in simple biochemical networks, Nature 387 (1997) 913.

[86] J. Stelling, U. Sauer, Z. Szallasi, F.J. Doyle, J. Doyle, Robustness of cellular

functions, Cell, 118 (2004) 675 .
[87] B. Novak, J.J. Tyson, Modelling the controls of the eukaryotic cell cycle, Biochem. Soc. Trans. 31 (2003) 1526.

[88] J.E. Ferrel, W. Xiong, Bistability in cell signaling: How to make continuous processes discontinuous, and reversible processes irreversible, Chaos, 11 (2001) 227. [89] T.Bondeva, L. Pirola, G. Bulgarelli-Leva, I. Rubio, R. Wetzker, M.P. Wymann, Bifurcation of lipid and protein kinase signals of P13K $\gamma$ to the protein kinases PKB and MAPK, Science 282 (1998) 293.

[90] J.S.Ferreira, R. Lozano, S. Mondie, A. Friboulet, Bifurcation analysis of a biochemical network, In Positive System, LNCIS 341, C. Commault, N. Merchand (Eds.), (2006) 279.

[91] V.N. Belykh, I.V. Belykh, M.Colding-Jorgensen, E. Mosekilde, Homoclinic bifurcations leading to the emergence of bursting oscillations in cell models, Eur. Phs. J. E 3 (2000) 205.

[92] A.M. Robert, C.S. Robert, L. Robert, Symmetry breaking in biological systems. From molecules to tissues, Struct. Chem. 18 (2007) 899.

[93] R.J. Sadus, Molecular simulation and theory for nanosystems: Insights for molecular motors, Mol. Sim. 34 (2008) 23.

[94] D.T. Gillespie, Stochastic simulation of chemical kinetics, Annu. Rev. Phys. Chem. 58 (2207) 35.

[95] B.A. Beard, H. Qian, Chemical Bophysics: Quantitative Analysis of Cellular Systems, Cambridge University Press, London, 2008.

[96] C. Antoine, A. Lemarchand, Resonance of relaxation time in the temperature modulated Schlögl model, J. Chem. Phys. 126 (2007) 104103.

[97] O. Aono, Thermodynamic coupling of diffusion with chemical reaction, J. Stat. Phys. 13 (1975) 331.

[98] E.M. Sevick, R. Prabhakar, S.R. Williams, D.J. Searles, Fluctuation theorems, Annu. Rev. Phys. Chem. 59 (2008) 603.

[99] B. Han, J. Wang, Least dissipation cost as a design principle for robustness and function of cellular networks, Phys. Rev. E 77 (2008) 031922.

[100] F. Schlögl, Chemical reaction models for nonequilibrium phase transitions, Z. Physik, 253 (1972) 1247

[101] J.O. de Zerate, J.V. Sengers, Hydrodynamic Fluctuations in Fluids and Fluid [102] H. Ge, H. Qian, Thermodynamic limit of a nonequilibrium steady state: Maxwell-type construction for a bistable biochemical network, Phys. Rev. Let. 103 (2009) 148103-1.

[103] D.-Q. Jiang, M. Qian, M.-P. Qian, Mathematical Theory of Nonequilibrium Steady States, Lect. Notes Math. Vol. 1833, Springer-Verlag, Berlin, 2004

[104] M. Vellela, H. Qian, A quasistationary analysis of a stochastic chemical reaction: Keizer's paradox, Bull. Math. Biol. 69 (2007) 1727.

[105] R.C. Dewar, Maximum entropy production and the fluctuation theorem, J. Phys. A Math. Gen. 38 (2005) L371.

[106] M. Stein, R.R. Gabdoulline, R.C. Wade, Bridging from molecular simulation to biochemical networks, Curr. Opinion Struc. Biol. 17 (2007) 166.

[107] D. Parker, Z. Bryant, S.L. Delp, Coarse-grained structural modeling of molecular motors using multibody dynamics, Cell. Mol. Bioeng. 2 (2009) 366.

[108] J.A. Papin, N.D. Price, B.Ø. Palsson, Extreme pathway lengths and reaction participation in genome-scale metabolic networks, Genome Res. 12 (2002) 1889.

[109] Q. Huang, H. Qian, Ultrasensitive dual phorphorylation dephosphorylation cycle kinetics exhibits canonical competition behavior, Chaos 19 (2009) 033109-1.

[110] W.J. Heuett, H. Qian, Grand canonical Markov model: a stochastic theory for open nonequilibrium biochemical networks, J. Chem. Phys. 124 (2006) 044110.

[111] H. Qian, E.L. Elson, Single-molecule enzymology: stochastic MichaelisMenten kinetics, Biophys. Chem. 101 (2002) 565.

[112] J.C. Liao, Custom design of metabolism, Nature Biotechnol. 22 (2004) 823.

[113] G.V. de Vijver, Self-Organization and Emergence in Life Sciences, Springer, Dordrecht, 2006.
[114] H. Qian, A simple theory of motor protein kinetics and energetic II, Biophys. Chem. 83 (2000) 35.

115] H. Qian, The mathematical theory of molecular motor movement and chemomechanical energy transduction, J. Math. Chem. 27 (2000) 219.

[116] H. Qian, Equations for stochastic macromolecular mechanics of single proteins: equilibrium fluctuations, transient kinetics, and nonequilibrium steady state, J. Phys. Chem. B 106 (2002) 2065.

[117] J.E. Baker, Free energy transduction in a chemical motor, J. Theor. Biol. 228

(2004) 467.

119] F. Nédélec, Computer simulations reveal motor properties generating stable

[120] P. Reimann, P. Hänggi, Introduction to the physics of Brownian motors, Appl. Phys. Appl. Phys. A 75 (2002) 169.

[121] J.E. Baker, C. Brosseau, P.B. Joel, D.M. Warshaw, The biochemical kinetics underlying actin movement generated by one and many skeletal muscle myosin molecules, Biophys. J. 82, (2002) 2134.

[122] W. Wiechert, The thermodynamic meaning of metabolic exchange fluxes, Biophys. J. 93 (2007) 2255

[123] G.E. Crooks, Entropy production fluctuation theorem and the nonequilibrium work relation for free energy differences, Phys. Rev. E 60 (1999) 2721.

[124] D. Collin, F. Ritort, C. Jarzynski, S.B. Smith, I. Tinoco, Jr, C. Bustamante, Verification of the Crooks fluctuation theorem and recovery of RNA folding free energies, Nature 437 (2005) 231.

[125] P. Turina, D. Samurai, P. Graber, H+/ATP ratio of proton transport-coupled ATP synthesis and hydrolysis catalyzed by CF0F1 tliposomes, Eur. Mol. Biology Org. J. 22 (2003) 418.

[126] Y. Sambongi, I. Ueda, Y. Wada, M. Futai, A biological molecular motor, proton translocating ATP synthase: multidiciplininary approach for a unique membrane enzyme, J. Bioenerg. Biomembr. 32 (2000) 441.

[127] M.Tsumuraya, S. Furuike, K. Adachi, K. Kinosita Jr., M. Yoshida, Effect of $\varepsilon$ subunit on the rotation of thermophilic Bacillus $\mathrm{F}_{1}$-ATPase, FEBS Letters, $\underline{583}$ (2009) 1121.

[128] P. Gaspard, Fluctuation Theorem, nonequilibrium work, and molecular machines, Proceedings of the $21^{\text {st }}$ Solvay Conference on Chemistry, Brussels, Nov. 28-Dec. $1^{\text {st }}(2008)$

[129] K. Shiroguchi, K. Kinosita, Myosin V Walks by lever action and Brownian motion, Science 316 (2007) 1208.

[130] M. Bier, Accounting for the energies and entropies of kinesin as catalytic cycle Eur. Phys. J. B 65 (2008) 415.

[131] E.A. Calzetta, Kinesin and the Crooks fluctuation theorem, Eur. Phys. J. B 68 (2009) 601.

[132] J. Castresana, Comparative genomics and bioenergetics, Biochim. Biophys. Acta 1506 (2001) 147.

[133] J.W. Stucki, The optimal efficiency and the economic degrees of coupling of oxidative phosphorylation, Euro. J. Biochem. 109 (1980) 269.

[134] C.B. Cairns, J. Walther, A.L. Harken, A. Banerjee, Mitochondrial oxidative phosphorylation thermodynamic efficiencies reflect physiological organ roles, Am. J. Physiol. Regul. Integr. Comp. Physiol. 433 (1998) R1376.

[135] O. Kedem, S.R. Caplan, Degree of coupling and its relation to efficiency in energy conversion, Trans. Faraday Soc. 61 (1965) 1897.

[136] D. Andrieux, P. Gaspard, Entropy production and time asymmetry in nonequilibrium fluctuation, Phys. Rev. Let. 98 (2007) 150601-1.

[137] Y. Demirel, Exergy use in bioenergetics, Int. J. Exergy, 1 (2004) 128.

[138] Y. Demirel, Information and living systems in 'The Ashgate Companion to Contemporary Philosophy of Biology,' Eds. G. Terzis, R. Arp, MIT Press, Cambridge, 2010.

[139]S. Cortes, N. Glade, I. Chartier, J. Tabony, Microtubule self-organisation by reaction-diffusion processes in miniature cell-sized containers and phospholipid vesicles, Biophys. Chem. 120 (2006) 168

[140] A. Turing, The chemical basis of morphogenesis, Philos. Trans. R. Soc. Lond. B Biol. Sci. $237(1952) 37$.

[141] V.V. Castets, E. Dulos, J. Boissonade, P. De Kepper, Experimental evidence of a sustained standing Turing-type nonequilibrium chemical pattern, Phys. Rev. Lett. 64 (1990) 2953.

[142] D.E. Strier, S.P. Dawson, Turing patterns inside cells, PLoS ONE 10 (2007) 1 [143] P.K. Maini, R.E. Baker, C.M. Chuong, Developmental biology. The Turing model comes of molecular age, Science 314 (2006) 1397.

[144] S.M. Mercer, J.M. Banks, D.L. Leaist, Nernst-Planck analysis of propagating reaction diffusion fronts in the aqueous iodate-arsenous acid system, Phys. Chem. Chem. Phys. 9 (2007) 5457.

[145] K.B. Oldham, J.C. Myland, Fundamentals of Electrochemical Science, Academic, New York, 1994

[146] Y. Demirel, Modeling of thermodynamically coupled reaction-transport systems, Chem. Eng. J. 139 (2008) 106.

[147] Y. Demirel, S.I. Sandler, Linear non-equilibrium thermodynamics theory for coupled heat and mass transport, Int. J. Heat Mass Transfer 44 (2001) 2439. 